\title{
Nonstandard neutrino interactions at DUNE, T2HK and T2HKK
}

\author{
Jiajun Liao, $^{a}$ Danny Marfatia ${ }^{a}$ and Kerry Whisnant ${ }^{b}$ \\ ${ }^{a}$ Department of Physics and Astronomy, University of Hawaii-Manoa, \\ Honolulu, HI 96822, U.S.A. \\ ${ }^{b}$ Department of Physics and Astronomy, Iowa State University, \\ Ames, IA 50011, U.S.A. \\ E-mail: liaoj@hawaii.edu, dmarf8@hawaii.edu, whisnant@iastate.edu
}

\begin{abstract}
We study the matter effect caused by nonstandard neutrino interactions (NSI) in the next generation long-baseline neutrino experiments, DUNE, T2HK and T2HKK. If multiple NSI parameters are nonzero, the potential of these experiments to detect CP violation, determine the mass hierarchy and constrain NSI is severely impaired by degeneracies between the NSI parameters and by the generalized mass hierarchy degeneracy. In particular, a cancellation between leading order terms in the appearance channels when $\epsilon_{e \tau}=\cot \theta_{23} \epsilon_{e \mu}$, strongly affects the sensitivities to these two NSI parameters at T2HK and T2HKK. We also study the dependence of the sensitivities on the true CP phase $\delta$ and the true mass hierarchy, and find that overall DUNE has the best sensitivity to the magnitude of the NSI parameters, while T2HKK has the best sensitivity to CP violation whether or not there are NSI. Furthermore, for T2HKK a smaller off-axis angle for the Korean detector is better overall. We find that due to the structure of the leading order terms in the appearance channel probabilities, the NSI sensitivities in a given experiment are similar for both mass hierarchies, modulo the phase change $\delta \rightarrow \delta+180^{\circ}$.
\end{abstract}

Keywords: Beyond Standard Model, CP violation, Neutrino Physics

ARXIV EPRINT: 1612.01443 


\section{Contents}

1 Introduction 1

2 Experiments 3

2.1 Experimental configurations 3

2.2 Simulation details 4

3 Sensitivities to NSI parameters and CP violation $\quad 6$

$\begin{array}{lll}3.1 & \text { Oscillation probabilities } & 6\end{array}$

3.2 NSI in the appearance channels $\left(\epsilon_{e \mu}, \epsilon_{e \tau}\right.$ and $\left.\epsilon_{e e}\right) \quad 7$

$\begin{array}{lll}3.2 .1 & \text { A single nonzero NSI parameter } & 7\end{array}$

$\begin{array}{lll}3.2 .2 & \text { Three nonzero NSI parameters } & 9\end{array}$

$\begin{array}{ll}3.2 .3 & \text { Dependence of the sensitivity on } \delta \\ 3\end{array}$

$\begin{array}{ll}\text { 3.2.4 Sensitivity when the true mass hierarchy is inverted } & 15\end{array}$

$\begin{array}{lll}3.3 & \text { NSI in the disappearance channels }\left(\epsilon_{\mu \tau} \text { and } \epsilon_{\tau \tau}\right) & 19\end{array}$

$\begin{array}{lll}4 & \text { Summary } & 20\end{array}$

\section{Introduction}

The success of neutrino oscillation experiments in the last few decades is a significant triumph in modern physics, and the masses and mixing angles of neutrinos have been incorporated into the standard model (SM) [1]. The data from a plethora of neutrino experiments using solar, atmospheric, reactor, and accelerator neutrinos can be explained in the framework of three neutrino mixing, in which the three known neutrino flavor eigenstates $\left(\nu_{e}, \nu_{\mu}, \nu_{\tau}\right)$ are quantum superpositions of three mass eigenstates $\left(\nu_{1}, \nu_{2}, \nu_{3}\right)$. In the SM with three massive neutrinos, the neutrino oscillations probabilities are determined by six oscillation parameters: two mass-squared differences $\left(\delta m_{21}^{2}, \delta m_{31}^{2}\right)$, three mixing angles $\left(\theta_{12}, \theta_{13}, \theta_{23}\right)$ and one Dirac CP phase $\delta$. Currently, the first five oscillation parameters have been well determined (up to the sign of $\delta m_{31}^{2}$ ) to the few percent level, and the main physics goals of current and future neutrino experiments are to measure the Dirac CP phase and to determine the neutrino mass hierarchy (MH), i.e., the sign of $\delta m_{31}^{2}$, and the octant of $\theta_{23}$, i.e., whether $\theta_{23}$ is larger or smaller than $45^{\circ}$. Future neutrino oscillation experiments will reach the sensitivity to do precision tests of the three neutrino oscillation paradigm and probe new physics beyond the SM.

A model-independent way of studying new physics in neutrino oscillation experiments is provided by the framework of nonstandard interactions (NSI); for recent reviews see refs. $[2,3]$. In this framework, new physics is parametrized as NSI at production, detection and in propagation according to their effects on the experiments. Since model-independent 
bounds on the production and detection NSI are generally an order of magnitude stronger than the matter NSI [4], we neglect production and detection NSI in this work, and focus on matter NSI, which can be described by dimension-six four-fermion operators of the form $[5,6]$

$$
\mathcal{L}_{\mathrm{NSI}}=2 \sqrt{2} G_{F} \epsilon_{\alpha \beta}^{\mathfrak{f} C}\left[\bar{\nu}_{\alpha} \gamma^{\rho} P_{L} \nu_{\beta}\right]\left[\overline{\mathfrak{f}} \gamma_{\rho} P_{C} \mathfrak{f}\right]+\text { h.c. },
$$

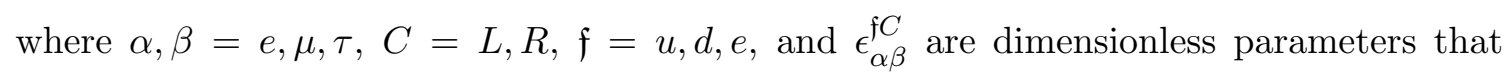
quantify the strength of the new interaction in units of $G_{F}$.

The Hamiltonian for neutrino propagation in the presence of matter NSI can be written as

$$
H=\frac{1}{2 E}\left[U\left(\begin{array}{ccc}
0 & 0 & 0 \\
0 & \delta m_{21}^{2} & 0 \\
0 & 0 & \delta m_{31}^{2}
\end{array}\right) U^{\dagger}+V\right]
$$

where $U$ is the Pontecorvo-Maki-Nakagawa-Sakata mixing matrix [1]

$$
U=\left(\begin{array}{ccc}
c_{13} c_{12} & c_{13} s_{12} & s_{13} e^{-i \delta} \\
-s_{12} c_{23}-c_{12} s_{23} s_{13} e^{i \delta} & c_{12} c_{23}-s_{12} s_{23} s_{13} e^{i \delta} & c_{13} s_{23} \\
s_{12} s_{23}-c_{12} c_{23} s_{13} e^{i \delta} & -c_{12} s_{23}-s_{12} c_{23} s_{13} e^{i \delta} & c_{13} c_{23}
\end{array}\right),
$$

and $V$ represents the potential from interactions of neutrinos in matter,

$$
V=A\left(\begin{array}{ccc}
1+\epsilon_{e e} & \epsilon_{e \mu} e^{i \phi_{e \mu}} & \epsilon_{e \tau} e^{i \phi_{e \tau}} \\
\epsilon_{e \mu} e^{-i \phi_{e \mu}} & \epsilon_{\mu \mu} & \epsilon_{\mu \tau} e^{i \phi_{\mu \tau}} \\
\epsilon_{e \tau} e^{-i \phi_{e \tau}} & \epsilon_{\mu \tau} e^{-i \phi_{\mu \tau}} & \epsilon_{\tau \tau}
\end{array}\right)
$$

Here $c_{j k} \equiv \cos \theta_{j k}, s_{j k} \equiv \sin \theta_{j k}, A \equiv 2 \sqrt{2} G_{F} N_{e} E, N_{e}$ is the number density of electrons, the unit contribution to $V_{e e}$ arises from the standard charged-current interaction. The effective NSI parameters are given by

$$
\epsilon_{\alpha \alpha}=\sum_{\mathfrak{f}, C} \epsilon_{\alpha \alpha}^{\mathfrak{f}, C} \frac{N_{\mathfrak{f}}}{N_{e}} \quad \text { and } \quad \epsilon_{\alpha \beta} e^{i \phi_{\alpha \beta}}=\sum_{\mathfrak{f}, C} \epsilon_{\alpha \beta}^{\mathfrak{f}, C} \frac{N_{\mathfrak{f}}}{N_{e}}(\alpha \neq \beta),
$$

where $N_{\mathfrak{f}}$ is the number density for fermion $\mathfrak{f}$. In the earth, $N_{u} \simeq N_{d} \simeq 3 N_{e}$. The diagonal terms in $V$ are real, and since the neutrino oscillation probabilities are not affected by a subtraction of a term proportional to the identity matrix, one of the diagonal terms can be chosen to be 0 . The off-diagonal terms are in general complex.

Since neutral-current interactions affect neutrino propagation coherently, long-baseline neutrino experiments with a well-understood beam and trajectory are an ideal place to probe matter NSI. Studies of matter NSI effects in the MINOS experiment have been performed in refs. $[7,8]$ and by the MINOS collaboration $[9,10]$. NSI analyses related to the currently running $\mathrm{T} 2 \mathrm{~K}$ [11] and $\mathrm{NO} \nu \mathrm{A}$ [12] experiments can be found in refs. [13-18]. However, due to large systematic uncertainties and limited statistics, these experiments cannot make a definitive measurement of the matter NSI.

The next generation long-baseline neutrino experiments, DUNE (Deep Underground Neutrino Experiment) [19], T2HK (Tokai-to-Hyper-Kamiokande) [20] and T2HKK (Tokaito-Hyper-Kamiokande-and-Korea) [21] will collect much more data than the current experiments. With improved systematic uncertainties, these next generation experiments 


\begin{tabular}{|c|c|c|c|c|}
\hline Experiment & $\frac{L(\mathrm{~km})}{E_{\text {peak }}(\mathrm{GeV})}$ & $\begin{array}{l}\nu+\bar{\nu} \text { Exposure } \\
\left(\mathrm{kt} \cdot \mathrm{MW} \cdot 10^{7} \mathrm{~s}\right)\end{array}$ & $\begin{array}{l}\text { Signal } \\
\text { norm. } \\
\text { uncertainty }\end{array}$ & $\begin{array}{l}\text { Background } \\
\text { norm. } \\
\text { uncertainty }\end{array}$ \\
\hline $\begin{array}{l}\text { DUNE } \\
\text { (LAr) }\end{array}$ & $\frac{1300}{3.0}$ & $\begin{array}{l}264+264 \\
(80 \mathrm{GeV} \text { protons, } 1.07 \mathrm{MW} \\
\text { power, } 1.47 \times 10^{21} \mathrm{POT} / \mathrm{yr}, \\
40 \mathrm{kt} \text { fiducial mass, } \\
3.5+3.5 \mathrm{yr})\end{array}$ & $\begin{array}{l}\text { app: } 2.0 \% \\
\text { dis: } 5.0 \%\end{array}$ & $\begin{array}{l}\text { app: } 5-20 \% \\
\text { dis: } 5-20 \%\end{array}$ \\
\hline $\begin{array}{l}\mathrm{T} 2 \mathrm{HK} \\
(\mathrm{WC})\end{array}$ & $\frac{295}{0.6}$ & $\begin{array}{l}864.5+2593.5 \\
(30 \mathrm{GeV} \text { protons, } 1.3 \mathrm{MW} \\
\text { power, } 2.7 \times 10^{21} \mathrm{POT} / \mathrm{yr}, \\
0.19 \mathrm{Mt} \text { each tank, } \\
1.5+4.5 \mathrm{yr} \text { with } 1 \text { tank, } \\
1+3 \mathrm{yr} \text { with } 2 \text { tanks })\end{array}$ & $\begin{array}{l}\text { app: } 2.5 \% \\
\text { dis: } 2.5 \%\end{array}$ & $\begin{array}{l}\text { app: } 5 \% \\
\text { dis: } 20 \%\end{array}$ \\
\hline $\begin{array}{l}\text { T2HKK-1.5 } \\
\text { (WC) }\end{array}$ & $\frac{295}{0.6}+\frac{1100}{0.8}$ & \multirow{2}{*}{$\begin{array}{l}1235+3705 \\
(30 \mathrm{GeV} \text { protons, } 1.3 \mathrm{MW} \\
\text { power, } 2.7 \times 10^{21} \mathrm{POT} / \mathrm{yr}, \\
0.19 \mathrm{Mt} \text { each tank, } 2.5+7.5 \mathrm{yr} \\
\text { with } 1 \text { tank at } \mathrm{KD} \text { and } \mathrm{HK})\end{array}$} & \multirow[t]{2}{*}{$\begin{array}{l}\text { app: } 2.5 \% \\
\text { dis: } 2.5 \%\end{array}$} & \multirow[t]{2}{*}{$\begin{array}{l}\text { app: } 5 \% \\
\text { dis: } 20 \%\end{array}$} \\
\hline $\begin{array}{l}\text { T2HKK-2.5 } \\
\text { (WC) }\end{array}$ & $\frac{295}{0.6}+\frac{1100}{0.6}$ & & & \\
\hline
\end{tabular}

For DUNE, $1 \mathrm{yr}=1.76 \times 10^{7} \mathrm{~s} ;$ for HyperK, $1 \mathrm{yr}=1.0 \times 10^{7} \mathrm{~s}$.

Table 1. Comparison of the experiments considered in this work.

may reach the sensitivity to discover NSI in the neutrino sector. Studies of matter NSI at DUNE and T2HK can be found in refs. [18, 22-28]. Studies of NSI with a second detector in Korea, in addition to the Kamiokande detector, can be found in refs. [29, 30]. ${ }^{1}$

In this paper, we use the new detector configuration proposed in the Hyper-Kamiokande proposal [20] and the fluxes [32] provided by the Hyper-K collaboration to study the performance of the T2HK and T2HKK experiments in the presence of NSI, and compare their sensitivities with the sensitivity of the DUNE experiment. In section 2, we describe the experiments considered in this work. In section 3, we discuss the sensitivities to SM and NSI parameters in each experiment. We summarize our results in section 4.

\section{Experiments}

\section{$2.1 \quad$ Experimental configurations}

The main features of the three next generation long-baseline neutrino experiments we consider are summarized in table 1 and details are described below.

\footnotetext{
${ }^{1}$ In refs. [22-27, 29, 30], the mass hierarchy is assumed to be known. As noted in ref. [18], and explained in ref. [31], if $\epsilon_{e e}$ is $\mathcal{O}(1)$, the mass hierarchy cannot be determined at long-baseline experiments, which in turn strongly affects a determination of the CP phase.
} 
DUNE: The DUNE experiment sends neutrinos from Fermilab to the Homestake mine in South Dakota with a baseline of $1300 \mathrm{~km}$. We followed the DUNE CDR [19] that uses a 40 kton liquid argon (LAr) detector sitting on axis with respect to the beam direction. There is a range of beam design options and here we choose the optimized design, which provides a better sensitivity in the appearance channel than the reference design. The optimized design utilizes an $80 \mathrm{GeV}$ proton beam with a power of $1.07 \mathrm{MW}$, which corresponds to $1.47 \times 10^{21}$ protons on target (POT) per year. We assume 3.5 years of running time in both neutrino and antineutrino beam modes, which gives a total exposure of $300 \mathrm{kt} \cdot \mathrm{MW} \cdot \mathrm{years}$.

T2HK: The T2HK [20] experiment uses an upgraded $30 \mathrm{GeV}$ J-PARC beam with a power of $1.3 \mathrm{MW}$, which corresponds to $2.7 \times 10^{21}$ POT per year. The Hyper-K detector is located $295 \mathrm{~km}$ away from the source $2.5^{\circ}$ off-axis so that it detects a narrow band beam with an unoscillated spectrum peaked at $0.6 \mathrm{GeV}$. Of the three detector configurations in the Hyper-K design report [20], we choose the 2TankHK-staged configuration, which has one tank taking data for 6 years and a second tank is added for another 4 years. Each tank has $40 \%$ photocoverage and contains a water Cherenkov (WC) detector with 0.19 Mton fiducial mass. We assume the running times between neutrino and antineutrino modes have a $1: 3$ ratio.

T2HKK: The T2HKK [21] experiment has one detector in the Kamioka mine, and a second detector in Korea. The Hyper-K detector (HK) is located in the same place as the T2HK experiment, with a $2.5^{\circ}$ off-axis-angle and a $295 \mathrm{~km}$ baseline. For the Korean detector (KD), we consider two options for the off-axis-angle: (a) T2HKK2.5 with the same $2.5^{\circ}$ off-axis-angle, and (b) T2HKK-1.5 with a $1.5^{\circ}$ off-axis-angle. Both KD options are at a baseline of $1100 \mathrm{~km}$. We assume the same neutrino beam as the T2HK experiment with an integrated beam power of $13 \mathrm{MW} \cdot y e a r s$, which corresponds to a total $2.7 \times 10^{22}$ POT. The total running time is 10 years with a ratio of $1: 3$ between neutrino and antineutrino modes.

\subsection{Simulation details}

We simulate the experiments using the GLoBES software [33, 34]. We use the official GLoBES simulation files released by the DUNE collaboration [35] which has the same experimental configurations as the DUNE CDR. The normalization uncertainties for the appearance and disappearance signal rates are $2 \%$ and $5 \%$, respectively. The background uncertainties are $5 \%$ except for the $\nu_{\tau}$ background, which has a $20 \%$ uncertainty. For the T2HK and T2HKK experiments, we matched the number of events reported in tables XXIX and XXX of ref. [20] in our simulation. We assume a normalization uncertainty of $2.5 \%$ for the signal rates, and $5 \%(20 \%)$ for the appearance (disappearance) background rates. Using the central values and uncertainties from a global fit in the SM scenario [36], we show the expected CP violation and mass hierarchy sensitivity of DUNE, T2HK, and T2HKK as a function of $\delta$ for both true normal and true inverted hierarchies in figures 1 and 2, respectively. From figure 1, we see that the expected CP violation sensitivities in our simulation are consistent with those in the DUNE [19] and Hyper-K [20] design reports. 


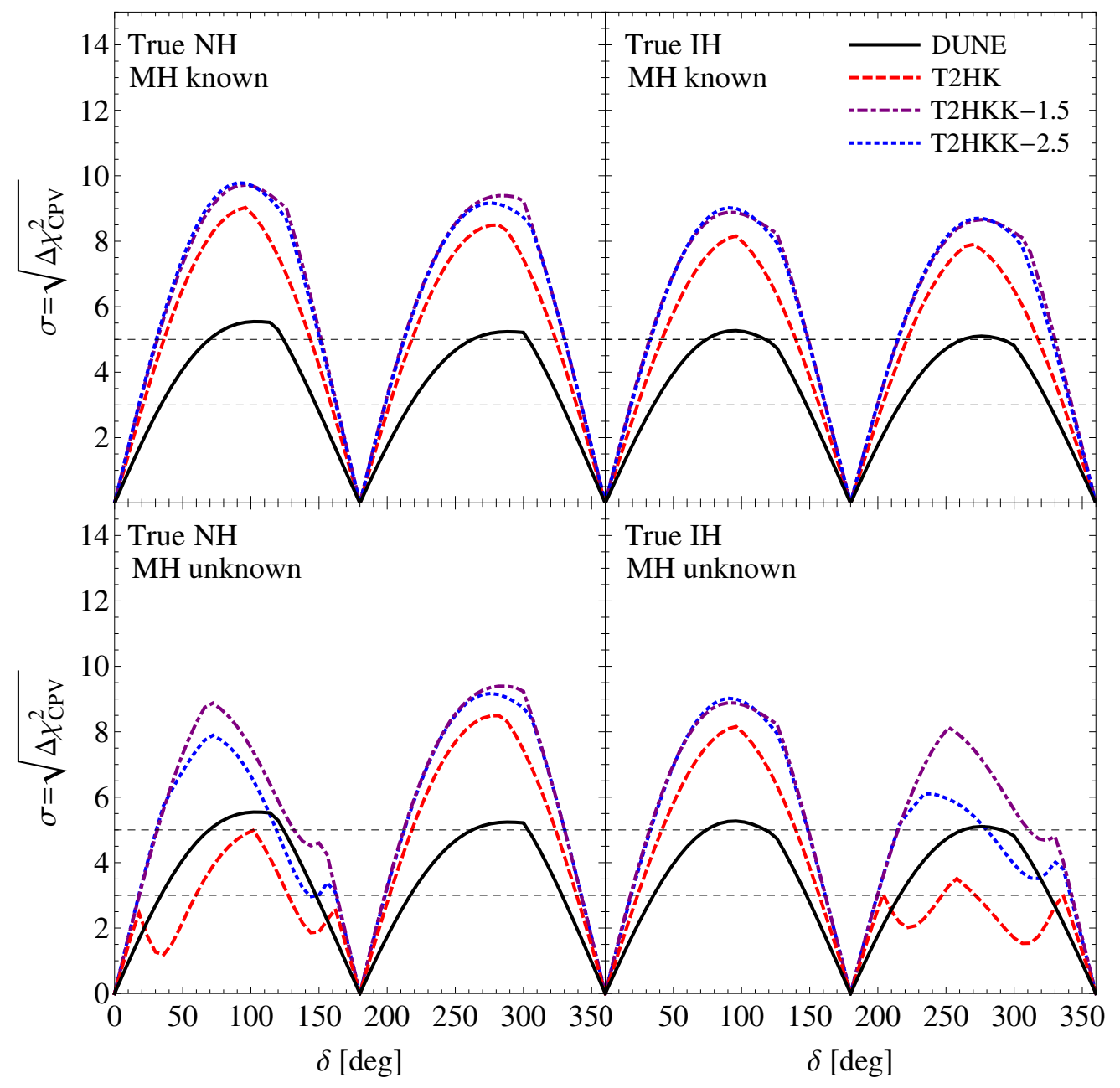

Figure 1. The expected sensitivity to CP violation as a function of SM $\delta$ at DUNE, T2HK, and T2HKK. The central values and uncertainties of the oscillation parameters are adopted from a global fit in the SM scenario [36], and a 5\% uncertainty for the matter density is assumed.

For the NSI scenario, we use the new physics tools developed in refs. [37, 38]. In our simulation, we use the Preliminary Reference Earth Model density profile [39] with a 5\% uncertainty for the matter density. ${ }^{2}$ The central values and uncertainties for the mixing angles and mass-squared differences are adopted from the global fit with NSI in ref. [40], which are

$$
\begin{aligned}
\sin ^{2} \theta_{13} & =0.023 \pm 0.002, \quad \sin ^{2} \theta_{23}=0.43_{-0.03}^{+0.08} \\
\sin ^{2} \theta_{12} & =(0.305 \pm 0.015) \oplus(0.70 \pm 0.017) \\
\delta m_{21}^{2} & =(7.48 \pm 0.21) \times 10^{-5} \mathrm{eV}^{2}, \quad\left|\delta m_{31}^{2}\right|=(2.43 \pm 0.08) \times 10^{-3} \mathrm{eV}^{2}
\end{aligned}
$$

\footnotetext{
${ }^{2}$ Note that in the DUNE CDR [35] a $2 \%$ uncertainty is used for the matter density, while a $6 \%$ uncertainty is used in the T2HK [20] and T2HKK [21] reports.
} 


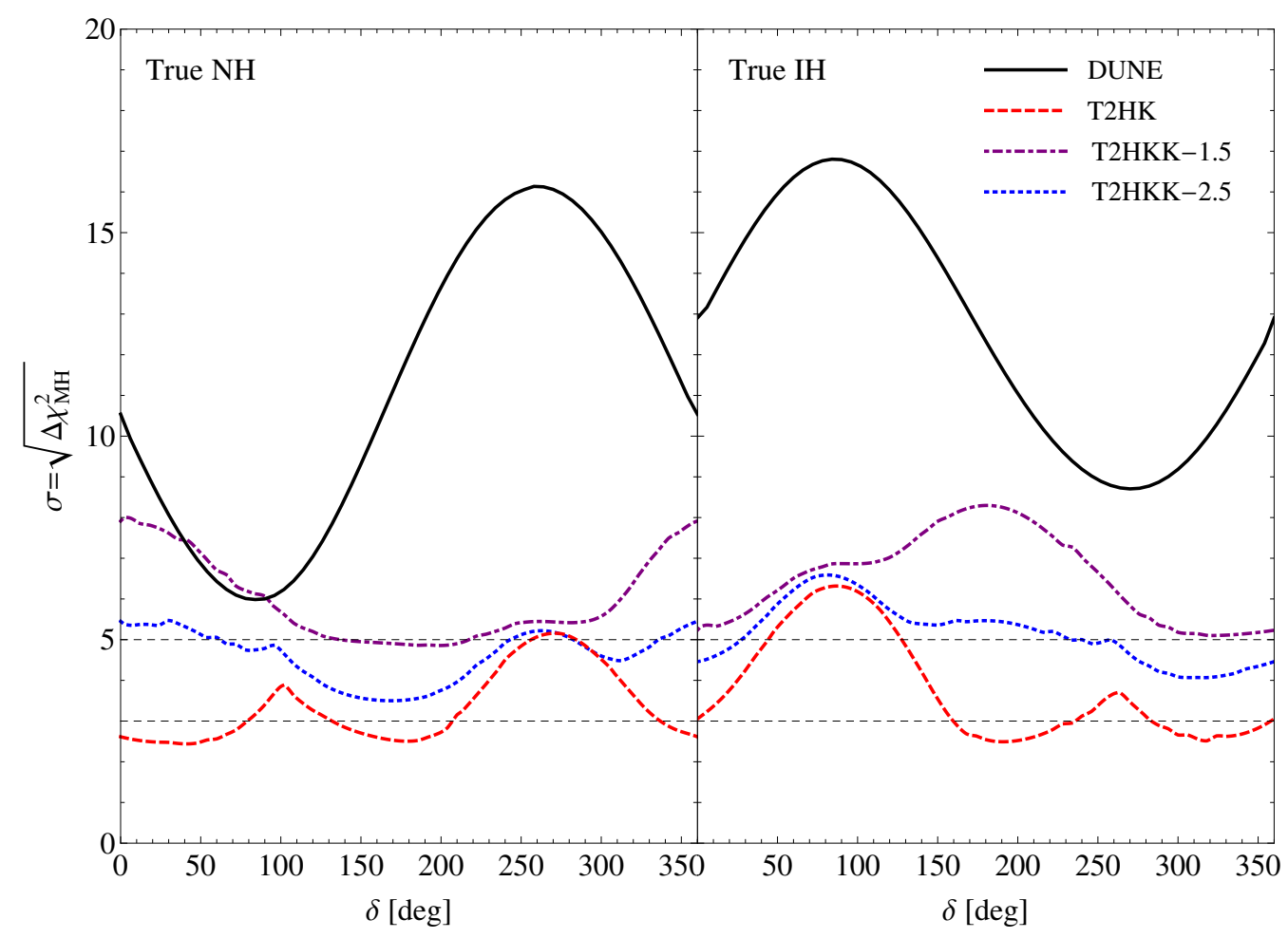

Figure 2. The expected sensitivity to the mass hierarchy as a function of SM $\delta$ at DUNE, T2HK, and T2HKK. The inputs and assumptions are the same as in figure 1.

For the NSI parameters, we scan over the following ranges suggested by the analysis of ref. [40],

$$
\begin{aligned}
& -5.0<\epsilon_{e e}<5.0, \quad \epsilon_{e \mu}<0.5, \quad \epsilon_{e \tau}<1.2, \\
& -0.6<\epsilon_{\tau \tau}<0.6, \quad \epsilon_{\mu \tau}<0.1,
\end{aligned}
$$

and marginalize over all the NSI phases in our simulation.

\section{Sensitivities to NSI parameters and CP violation}

\subsection{Oscillation probabilities}

The appearance probability for the normal hierarchy (NH) can be written as [18]

$$
\begin{aligned}
P\left(\nu_{\mu} \rightarrow \nu_{e}\right)= & x^{2} f^{2}+2 x y f g \cos (\Delta+\delta)+y^{2} g^{2} \\
& +4 \hat{A} \epsilon_{e \mu}\left\{x f\left[s_{23}^{2} f \cos \left(\phi_{e \mu}+\delta\right)+c_{23}^{2} g \cos \left(\Delta+\delta+\phi_{e \mu}\right)\right]\right. \\
& \left.+y g\left[c_{23}^{2} g \cos \phi_{e \mu}+s_{23}^{2} f \cos \left(\Delta-\phi_{e \mu}\right)\right]\right\} \\
& +4 \hat{A} \epsilon_{e \tau} s_{23} c_{23}\left\{x f\left[f \cos \left(\phi_{e \tau}+\delta\right)-g \cos \left(\Delta+\delta+\phi_{e \tau}\right)\right]\right. \\
& \left.-y g\left[g \cos \phi_{e \tau}-f \cos \left(\Delta-\phi_{e \tau}\right)\right]\right\} \\
& +4 \hat{A}^{2}\left(g^{2} c_{23}^{2}\left|c_{23} \epsilon_{e \mu}-s_{23} \epsilon_{e \tau}\right|^{2}+f^{2} s_{23}^{2}\left|s_{23} \epsilon_{e \mu}+c_{23} \epsilon_{e \tau}\right|^{2}\right) \\
& +8 \hat{A}^{2} f g s_{23} c_{23}\left\{c_{23} \cos \Delta\left[s_{23}\left(\epsilon_{e \mu}^{2}-\epsilon_{e \tau}^{2}\right)+2 c_{23} \epsilon_{e \mu} \epsilon_{e \tau} \cos \left(\phi_{e \mu}-\phi_{e \tau}\right)\right]\right. \\
& \left.-\epsilon_{e \mu} \epsilon_{e \tau} \cos \left(\Delta-\phi_{e \mu}+\phi_{e \tau}\right)\right\}+\mathcal{O}\left(s_{13}^{2} \epsilon, s_{13} \epsilon^{2}, \epsilon^{3}\right),
\end{aligned}
$$


where following ref. [41],

$$
\begin{aligned}
x & \equiv 2 s_{13} s_{23}, \quad y \equiv 2 r s_{12} c_{12} c_{23}, & r & =\left|\delta m_{21}^{2} / \delta m_{31}^{2}\right|, \\
f, \bar{f} & \equiv \frac{\sin \left[\Delta\left(1 \mp \hat{A}\left(1+\epsilon_{e e}\right)\right)\right]}{\left(1 \mp \hat{A}\left(1+\epsilon_{e e}\right)\right)}, & g & \equiv \frac{\sin \left(\hat{A}\left(1+\epsilon_{e e}\right) \Delta\right)}{\hat{A}\left(1+\epsilon_{e e}\right)}, \\
\Delta & \equiv\left|\frac{\delta m_{31}^{2} L}{4 E}\right|, & \hat{A} & \equiv\left|\frac{A}{\delta m_{31}^{2}}\right| .
\end{aligned}
$$

Henceforth we define $P_{\mu e} \equiv P\left(\nu_{\mu} \rightarrow \nu_{e}\right)$ and the antineutrino probability $\bar{P}_{\mu e} \equiv P\left(\bar{\nu}_{\mu} \rightarrow\right.$ $\bar{\nu}_{e}$ ), which is given by eq. (3.1) with $\hat{A} \rightarrow-\hat{A}$ (and hence $f \rightarrow \bar{f}$ ), $\delta \rightarrow-\delta$, and $\phi_{\alpha \beta} \rightarrow$ $-\phi_{\alpha \beta}$. For the inverted hierarchy (IH), $\Delta \rightarrow-\Delta, y \rightarrow-y, \hat{A} \rightarrow-\hat{A}$ (i.e., $f \leftrightarrow-\bar{f}$, and $g \rightarrow-g$ ). Our result agrees with the $\mathcal{O}(\epsilon)$ expressions in refs. [37, 42].

From eq. (3.1), we see that $\epsilon_{\mu \mu}, \epsilon_{\mu \tau}$ and $\epsilon_{\tau \tau}$ do not appear in the appearance probability up to second order in $\epsilon$. Hence, they mainly affect the disappearance channel. Taking $\epsilon_{e e}$, $\epsilon_{e \mu}$ and $\epsilon_{e \tau}$ equal to zero, the disappearance probability can be written as

$$
\begin{aligned}
P\left(\nu_{\mu} \rightarrow \nu_{\mu}\right)= & 1-\sin ^{2} 2 \theta_{23} \sin ^{2} \Delta+r c_{12}^{2} \sin ^{2} 2 \theta_{23} \Delta \sin 2 \Delta-\frac{4 s_{23}^{4} s_{13}^{2} \sin ^{2}(1-\hat{A}) \Delta}{(1-\hat{A})^{2}} \\
& -\frac{\sin ^{2} 2 \theta_{23} s_{13}^{2}}{(1-\hat{A})^{2}}[\hat{A}(1-\hat{A}) \Delta \sin 2 \Delta+\sin (1-\hat{A}) \Delta \sin (1+\hat{A}) \Delta] \\
& -2 \hat{A} \epsilon_{\mu \tau} \cos \phi_{\mu \tau}\left(\sin ^{3} 2 \theta_{23} \Delta \sin 2 \Delta+2 \sin 2 \theta_{23} \cos ^{2} 2 \theta_{23} \sin ^{2} \Delta\right) \\
& +\hat{A}\left(\epsilon_{\mu \mu}-\epsilon_{\tau \tau}\right) \sin ^{2} 2 \theta_{23} \cos 2 \theta_{23}\left(\Delta \sin 2 \Delta-2 \sin ^{2} \Delta\right) \\
& -2 \hat{A}^{2} \sin ^{2} 2 \theta_{23} \epsilon_{\mu \tau}^{2}\left(2 \sin ^{2} 2 \theta_{23} \cos ^{2} \phi_{\mu \tau} \Delta^{2} \cos 2 \Delta+\sin ^{2} \phi_{\mu \tau} \Delta \sin 2 \Delta\right) \\
& -\hat{A}^{2} \sin ^{4} 2 \theta_{23}\left(\epsilon_{\mu \mu}-\epsilon_{\tau \tau}\right)^{2}\left(\frac{1}{2} \Delta \sin 2 \Delta-\sin ^{2} \Delta\right) \\
& +\mathcal{O}\left(s_{13}^{2} \epsilon, r \epsilon, s_{13} \epsilon^{2}, \cos 2 \theta_{23} \epsilon^{2}, \epsilon^{3}\right) .
\end{aligned}
$$

Our result agrees with ref. [43] for the SM terms (in the first two lines) and with ref. [42] for the NSI terms up to second order after making the assumption that terms of order $\cos 2 \theta_{23} \epsilon^{2}$ can be ignored. Our result disagrees with ref. [27] in the second-order terms in $\epsilon$. We can see in eq. (3.3) that $\epsilon_{\mu \mu}$ and $\epsilon_{\tau \tau}$ appear in the form of their difference up to second order in $\epsilon$. We therefore choose $\epsilon_{\mu \mu}=0$.

\subsection{NSI in the appearance channels $\left(\epsilon_{e \mu}, \epsilon_{e \tau}\right.$ and $\left.\epsilon_{e e}\right)$}

We only consider $\epsilon_{e \mu}, \epsilon_{e \tau}$ and $\epsilon_{e e}$ in this section because $\epsilon_{\mu \mu}, \epsilon_{\mu \tau}$ and $\epsilon_{\tau \tau}$ do not appear in the appearance probabilities up to second order in $\epsilon$.

\subsubsection{A single nonzero NSI parameter}

For a single $L / E$, data consistent with the SM can be also described by a model with NSI if $P^{\mathrm{SM}}\left(\nu_{\mu} \rightarrow \nu_{e}\right)=P^{\mathrm{NSI}}\left(\nu_{\mu} \rightarrow \nu_{e}\right)$ and $P^{\mathrm{SM}}\left(\bar{\nu}_{\mu} \rightarrow \bar{\nu}_{e}\right)=P^{\mathrm{NSI}}\left(\bar{\nu}_{\mu} \rightarrow \bar{\nu}_{e}\right)$. Since the three mixing angles, $\delta m_{21}^{2}$ and $\left|\delta m_{31}^{2}\right|$ are well-measured by other experiments, if only one off-diagonal NSI $\left(\epsilon_{e \mu}\right.$ or $\left.\epsilon_{e \tau}\right)$ is nonzero, there exists a continuous four-fold degeneracy as 
a result of the unknown mass hierarchy and $\theta_{23}$ octant [18]. We denote $\delta\left(\delta^{\prime}\right)$ as the Dirac $\mathrm{CP}$ phase in the SM (NSI) scenario.

The continuous degeneracy can be understood as follows. If only one off-diagonal NSI is nonzero, there are three unknowns to be determined in the NSI scenario: $\delta^{\prime}$ (the Dirac CP phase in $P^{\mathrm{NSI}}$ ), the NSI magnitude $\epsilon$ and the NSI phase $\phi$. Since a single measurement of $P$ and $\bar{P}$ for a fixed $L$ and $E$ gives only two constraints, for each value of $\delta$ in the SM, a solution for $\epsilon$ and $\phi$ will exist for any value of $\delta^{\prime}$. This leads to continuous degeneracies throughout the two-dimensional $\delta$ - $\delta^{\prime}$ space. An additional measurement at a different $L$ and/or $E$ can be made to reduce the degeneracies to lines in $\delta-\delta^{\prime}$ space, i.e., for each value of $\delta$ there will only be one $\delta^{\prime}$ that will be degenerate. If there are multiple $\delta^{\prime}$ solutions, then a second additional measurement at a different $L$ and/or $E$ should in principle remove the degeneracies.

If only $\epsilon_{e e}$ is nonzero, since it is real, an experiment that measures $P$ and $\bar{P}$ at a single $L / E$ should be able to fix the SM value of $\delta$ and the NSI values of $\delta^{\prime}$ and $\epsilon_{e e}$. If a nontrivial solution exists, then there is a simple two-fold degeneracy between the SM and NSI, and at least one additional measurement is needed to break the degeneracy between the SM and NSI with $\epsilon_{e e}$. Note however that the nonlinearity (in $\epsilon_{e e}$ ) of the equations may yield several solutions with nonzero $\epsilon_{e e}$ and $\delta^{\prime} \neq \delta$.

Since DUNE, T2HK and T2HKK effectively measure probabilities at a variety of energies, in principle these experiments can not only resolve the degeneracies with NSI solutions, but also put severe restrictions on the NSI parameters. If only one NSI parameter is nonzero, the expected allowed regions in the $\delta^{\prime}-\epsilon_{e e},-\epsilon_{e \mu}$ and $-\epsilon_{e \tau}$ planes are shown in figures 3,4 and 5, respectively. We assume the data are consistent with the SM with $\delta=0$ and the NH. The results are obtained after scanning over both mass hierarchies.

From figure 3, we see that there is always an allowed region near $\epsilon_{e e}=-2$ and $\delta^{\prime}=$ $180^{\circ}$. This degeneracy at DUNE was first shown in ref. [18], and can be explained by the generalized MH degeneracy [31, 44], which states that under the transformation,

$$
\begin{array}{rlrl}
\delta m_{31}^{2} & \rightarrow-\delta m_{32}^{2}, & \theta_{12} & \rightarrow 90^{\circ}-\theta_{12}, \quad \delta \rightarrow 180^{\circ}-\delta, \\
\epsilon_{e e} & \rightarrow-\epsilon_{e e}-2, \quad \epsilon_{\alpha \beta} e^{i \phi_{\alpha \beta}} \rightarrow-\epsilon_{\alpha \beta} e^{-i \phi_{\alpha \beta}}(\alpha \beta \neq e e),
\end{array}
$$

the Hamiltonian transforms as $H \rightarrow-H^{*}$, and the oscillation probabilities are unchanged [31]. Since this degeneracy does not depend on $L$ and $E$, all long-baseline experiments, including atmospheric and reactor neutrino experiments (like JUNO [45]) cannot resolve this degeneracy if $\epsilon_{e e} \sim-2$.

From figure 4, we see that if only $\epsilon_{e \mu}$ is nonzero, the mass hierarchy degeneracy is resolved at DUNE and T2HKK. DUNE puts severe constraints on $\epsilon(\lesssim 0.15$ at $3 \sigma)$ while T2HKK-1.5 places better constraints on $\left|\delta^{\prime}\right|\left(\lesssim 30^{\circ}\right.$ at $\left.3 \sigma\right)$. However, T2HK cannot resolve the mass hierarchy in this case; the $\mathrm{IH}$ is still allowed for $\delta^{\prime} \sim 215^{\circ}$. Also, there is a $2 \sigma$ allowed region around $\epsilon_{e \mu} \sim 0.5$ arising from the $\theta_{23}$ octant degeneracy. Around this region, the second octant of $\theta_{23}$ for the IH has a smaller $\chi^{2}$ than the first octant.

If only $\epsilon_{e \tau}$ is nonzero, from figure 5 we see that the mass hierarchy is not resolved for any of the experiments, although the IH is not allowed at the $1 \sigma$ CL at DUNE. This could lead to a wrong determination of the Dirac CP phase in all the experiments. 


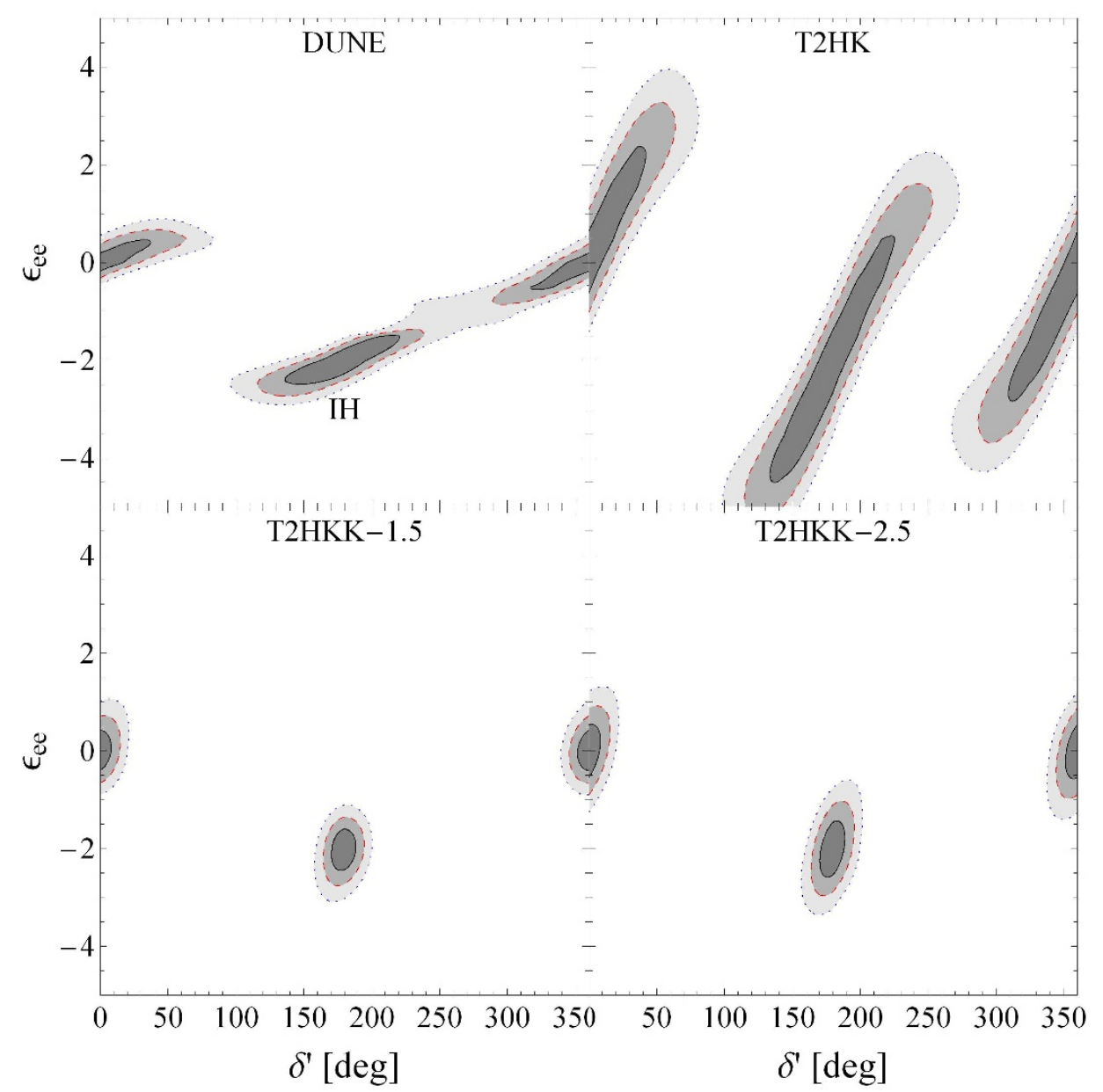

Figure 3. $1 \sigma, 2 \sigma$ and $3 \sigma$ allowed regions for $\epsilon_{e e}$ at DUNE, T2HK, and T2HKK when only $\epsilon_{e e}$ is nonzero. The data are consistent with the SM with $\delta=0$ and the NH. The allowed regions near $\delta^{\prime}=180^{\circ}$ are for the IH. Here $\delta\left(\delta^{\prime}\right)$ denotes the Dirac CP phase in the SM (NSI) scenario.

\subsubsection{Three nonzero NSI parameters}

If $\epsilon_{e \mu}, \epsilon_{e \tau}$ and $\epsilon_{e e}$ are all nonzero, then there are six free NSI parameters: $\delta^{\prime}, \epsilon_{e e}$, two magnitudes, and two phases. Even $P$ and $\bar{P}$ measurements at three different $L$ and $E$ combinations (six equations and six unknowns) could at most reduce the degeneracy to a single point in NSI parameter space (or perhaps a finite number of points). Therefore, an experiment that measures probabilities at a large variety of energies and/or distances is needed to resolve the degeneracies in the presence of multiple NSI.

The expected allowed regions in the $\delta^{\prime}-\epsilon_{e e},-\epsilon_{e \mu}$ and $-\epsilon_{e \tau}$ planes are shown in figures 6,7 and 8 , respectively. For each $\epsilon$, we scan over both $\mathrm{NH}$ and $\mathrm{IH}$, and marginalize over all the other NSI parameters. As expected, constraints on the NSI parameters become much worse. In particular, from figure 7 , we see that the constraint on $\epsilon_{e \mu}$ is much weaker at T2HK and T2HKK than at DUNE. This coincides with a strong degeneracy between 


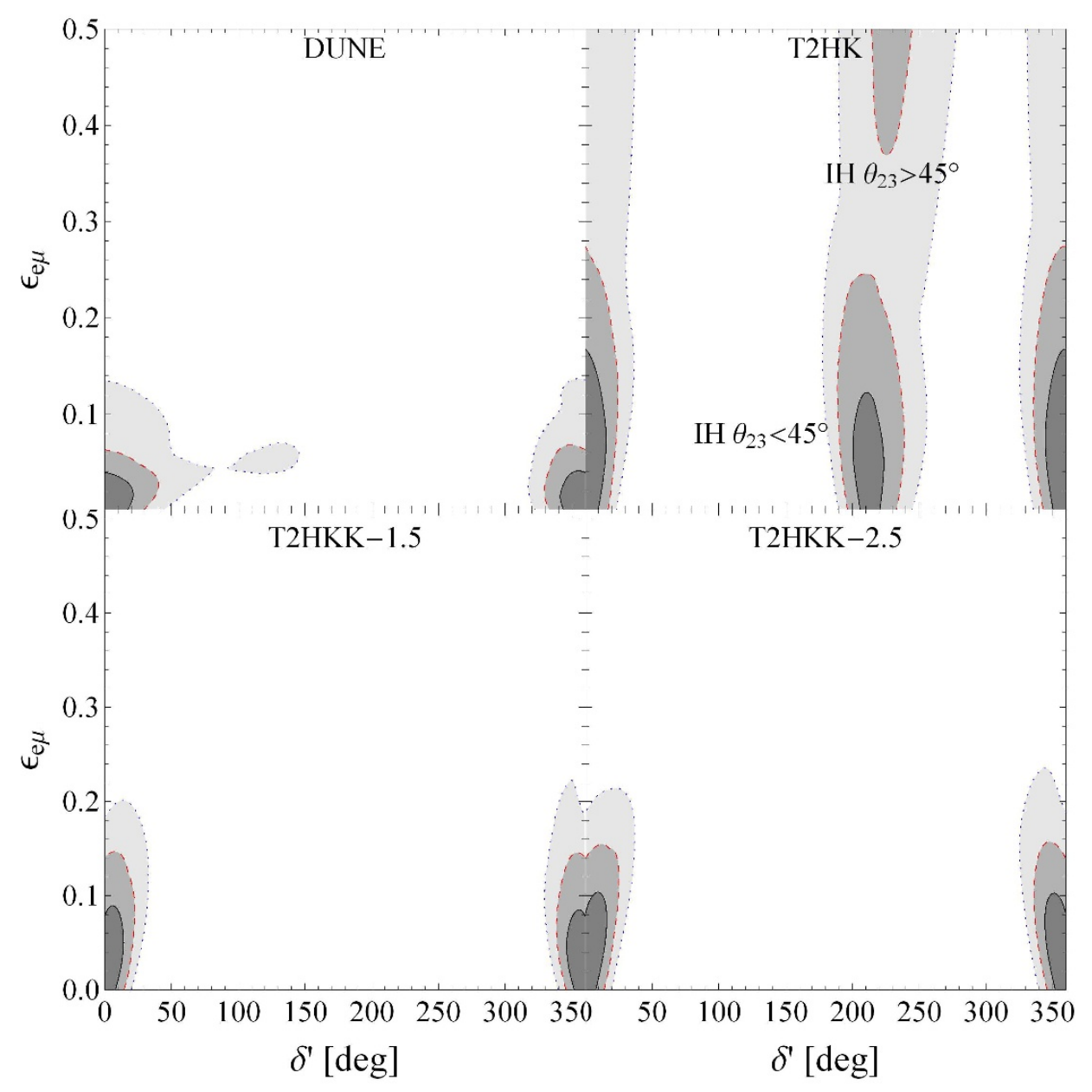

Figure 4. Same as figure 3, except for $\epsilon_{e \mu}$.

$\epsilon_{e \mu}$ and $\epsilon_{e \tau}$ at T2HK and T2HKK (see figure 9), and can be explained by examining the appearance probability in eq. (3.1).

For T2HK and T2HKK, since $\hat{A} \sim 0.05$ for $E \sim 0.6 \mathrm{GeV}$, the higher order terms from the matter effect can be neglected in eq. (3.1). Taking $\epsilon_{e e}=0$ and $\delta^{\prime}=\delta$, we have

$$
\begin{aligned}
P_{\mu e}^{\mathrm{NSI}}-P_{\mu e}^{\mathrm{SM}}= & 4 \hat{A} \epsilon_{e \mu} x f\left[s_{23}^{2} f \cos \left(\phi_{e \mu}+\delta\right)+c_{23}^{2} g \cos \left(\Delta+\delta+\phi_{e \mu}\right)\right] \\
& +4 \hat{A} \epsilon_{e \tau} x f\left[s_{23} c_{23} f \cos \left(\phi_{e \tau}+\delta\right)-s_{23} c_{23} g \cos \left(\Delta+\delta+\phi_{e \tau}\right)\right] \\
& +\mathcal{O}\left(y \hat{A} \epsilon, \hat{A}^{2} \epsilon^{2}\right) .
\end{aligned}
$$

If in addition, $\phi_{e \mu}=\phi_{e \tau}= \pm 90^{\circ}-\delta$, then

$$
P_{\mu e}^{\mathrm{NSI}}-P_{\mu e}^{\mathrm{SM}}=\mp 4 x f \hat{A} c_{23}\left(c_{23} \epsilon_{e \mu}-s_{23} \epsilon_{e \tau}\right) g \sin \Delta+\mathcal{O}\left(y \hat{A} \epsilon, \hat{A}^{2} \epsilon^{2}\right) .
$$

The corresponding equation for antineutrinos is given by $\hat{A} \rightarrow-\hat{A}, \delta \rightarrow-\delta$ and $\phi_{\alpha \beta} \rightarrow$ $-\phi_{\alpha \beta}$. Similar equations hold for the IH. As can be seen from eq. (3.6), if

$$
\epsilon_{e \tau}=\cot \theta_{23} \epsilon_{e \mu}
$$




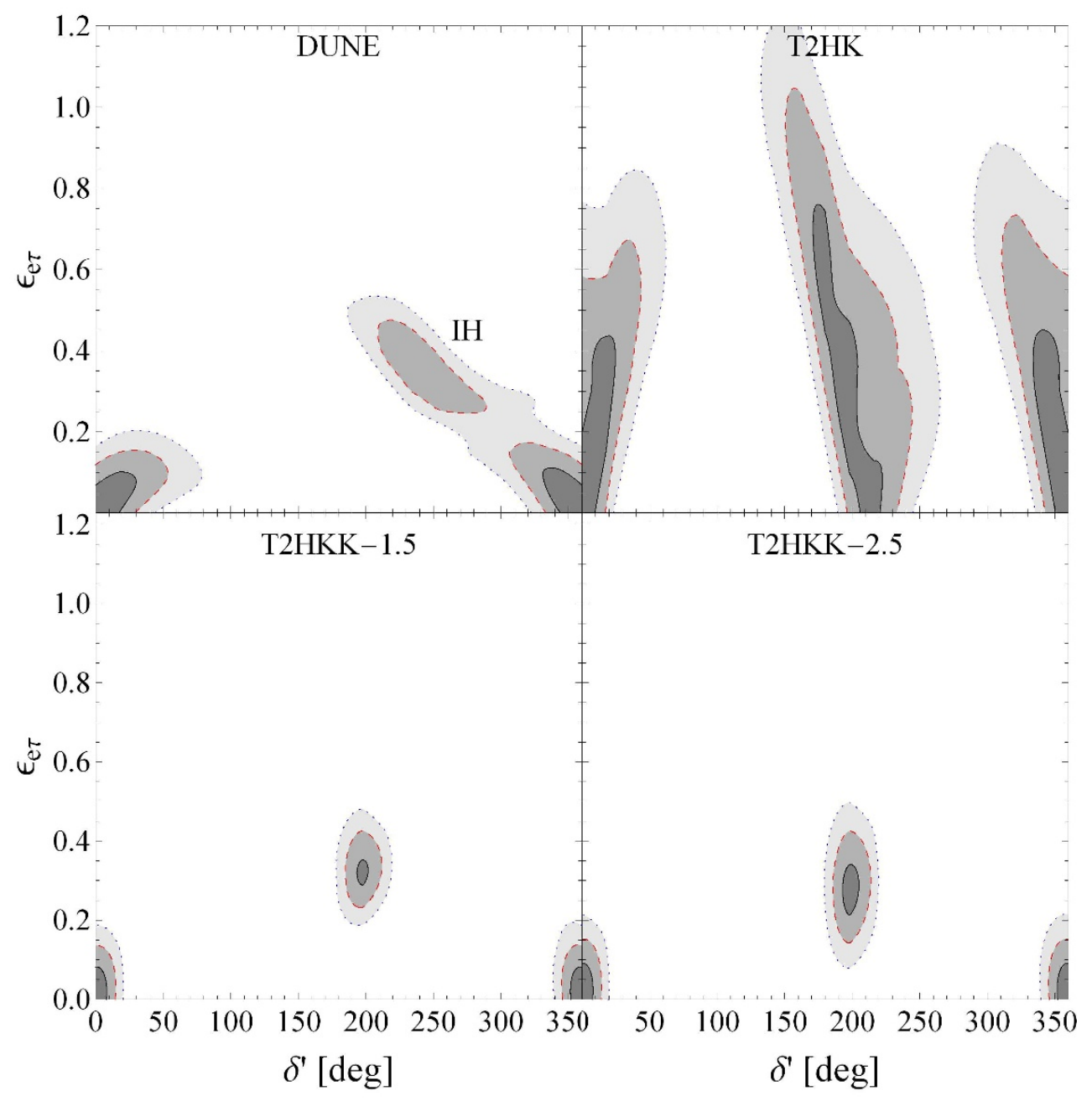

Figure 5. Same as figure 3, except for $\epsilon_{e \tau}$.

the difference between the NSI and SM appearance probabilities is strongly suppressed in both the neutrino and antineutrino modes. Consequently, the constraint on $\epsilon_{e \mu}$ is very weak at T2HK and T2HKK if $\epsilon_{e \tau} \simeq \cot \theta_{23} \epsilon_{e \mu}$. Since neutino energies at DUNE are much higher than at T2HK and T2HKK (e.g., $E_{\text {peak }} \sim 3 \mathrm{GeV}$ for which $\hat{A} \sim 0.28$ ), higher order terms in eq. (3.1) cannot be neglected, and the degeneracy between $\epsilon_{e \mu}$ and $\epsilon_{e \tau}$ can be resolved. Also, comparing the lower panels of figure 9 we see that T2HKK-1.5 starts to break the degeneracy between $\epsilon_{e \mu}$ and $\epsilon_{e \tau}$ for $\epsilon_{e \mu} \lesssim 0.5$ since T2HKK-1.5 has a higher peak energy than T2HKK-2.5.

We also find strong correlations between $\epsilon_{e \tau}$ and $\epsilon_{e e}$ in all experiments, which can be seen in figure 10. The allowed regions are symmetric around $\epsilon_{e e}=-1$ due to the generalized $\mathrm{MH}$ degeneracy; the vertex of the $\mathrm{V}$-shaped $\mathrm{NH}$ region is at $\epsilon_{e e}=0$ and vertex of the V-shaped IH region is at $\epsilon_{e e}=-2$. 


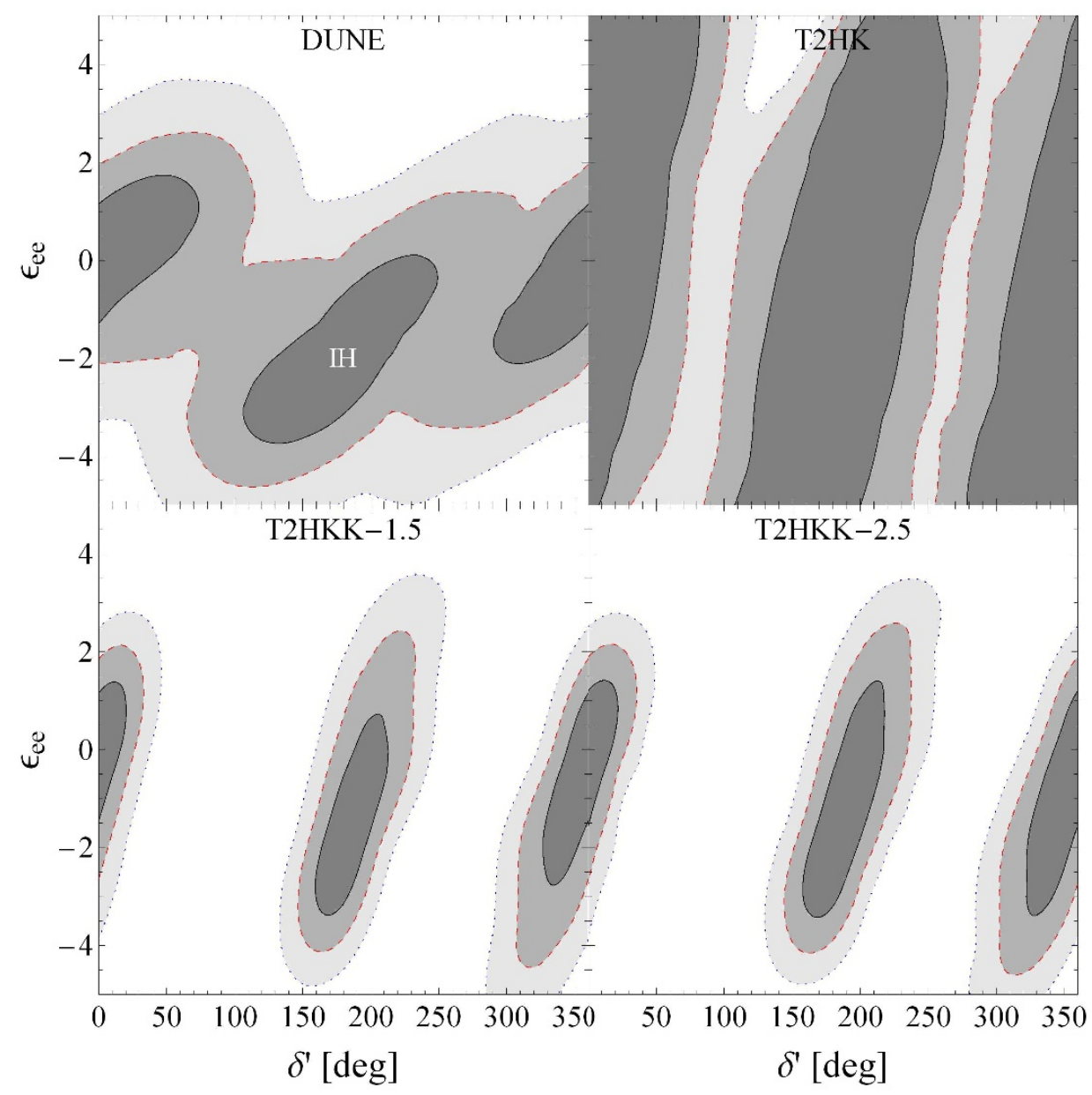

Figure 6. $1 \sigma, 2 \sigma$ and $3 \sigma$ allowed regions for $\epsilon_{e e}$ at DUNE, T2HK and T2HKK when $\epsilon_{e \mu}, \epsilon_{e \tau}$ and $\epsilon_{e e}$ are all nonzero. The data are consistent with the SM with $\delta=0$ and the NH. All other parameters not shown have been marginalized over.

\subsubsection{Dependence of the sensitivity on $\delta$}

Since both the Dirac CP phase $\delta$ and the mass hierarchy are unknown, the experimental performance may be affected by the true parameters in nature. In this section, we examine how the sensitivity changes with the true value of $\delta$. In the next section we study the sensitivity if the true hierarchy is inverted. Although the mass hierarchy will not be measured in neutrino oscillation experiments because of the generalized $\mathrm{MH}$ degeneracy, future neutrinoless double beta decay experiments may determine the mass hierarchy if neutrinos are Majorana particles. We therefore entertain the possibilities that the $\mathrm{MH}$ is known and that it is unknown.

We assume that the data are consistent with the SM and the NH, and plot the constraints on $\delta^{\prime}$ as a function of $\delta$ if all three $\epsilon$ 's are nonzero; see figures 11 and 12 for the case when mass hierarchy is known and unknown, respectively. We see that if the mass 


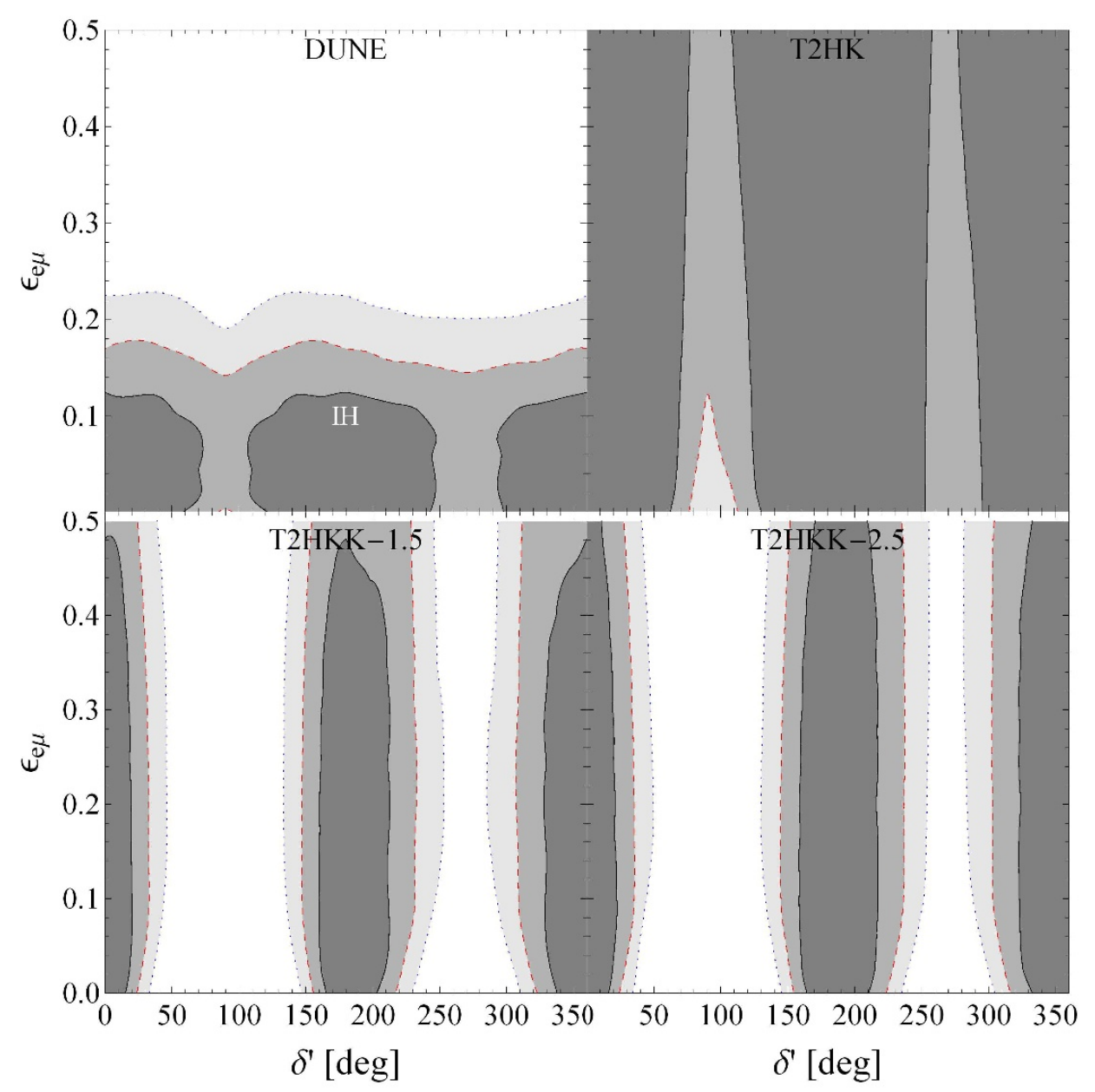

Figure 7. Same as figure 6, except for $\epsilon_{e \mu}$.

hierarchy is known, since $\delta^{\prime}=\delta$ always holds when $\epsilon=0$, the diagonal line in the $\delta^{\prime}$ versus $\delta$ plot is always allowed at less than $1 \sigma$. If the mass hierarchy is unknown, when the SM and NSI have the opposite mass hierarchy, there is a strong correlation between $\delta$ and $\delta^{\prime}$ (which can be described by $\delta^{\prime}=180-\delta$ ) as a result of the generalized MH degeneracy. We also see that T2HKK has a better performance than T2HK and DUNE in measuring $\delta$. In fact, if the mass hierarchy is unknown, only T2HKK can measure $\delta$ at the $3 \sigma$ CL when three $\epsilon$ 's are nonzero.

We also plot the minimum value of $\epsilon$ for which the NSI scenario can be discriminated from the SM at the $2 \sigma \mathrm{CL}$. If there is only one nonzero $\epsilon$, the expected sensitivities are shown in figures 13 and 14 if the $\mathrm{MH}$ is known and unknown, respectively. As expected, the sensitivity is always weaker if the $\mathrm{MH}$ is unknown than if the $\mathrm{MH}$ is known. Note that the minimum value of $\left|\epsilon_{e e}\right|$ that is detectable is always larger than 2 if the $\mathrm{MH}$ is unknown due to the generalized $\mathrm{MH}$ degeneracy. From figure 13 we see that the sensitivity to $\left|\epsilon_{e e}\right|$ and $\left|\epsilon_{e \tau}\right|$ at DUNE and T2HK improves for $\delta \simeq 90$ and $240^{\circ}$, while this is not the case for 


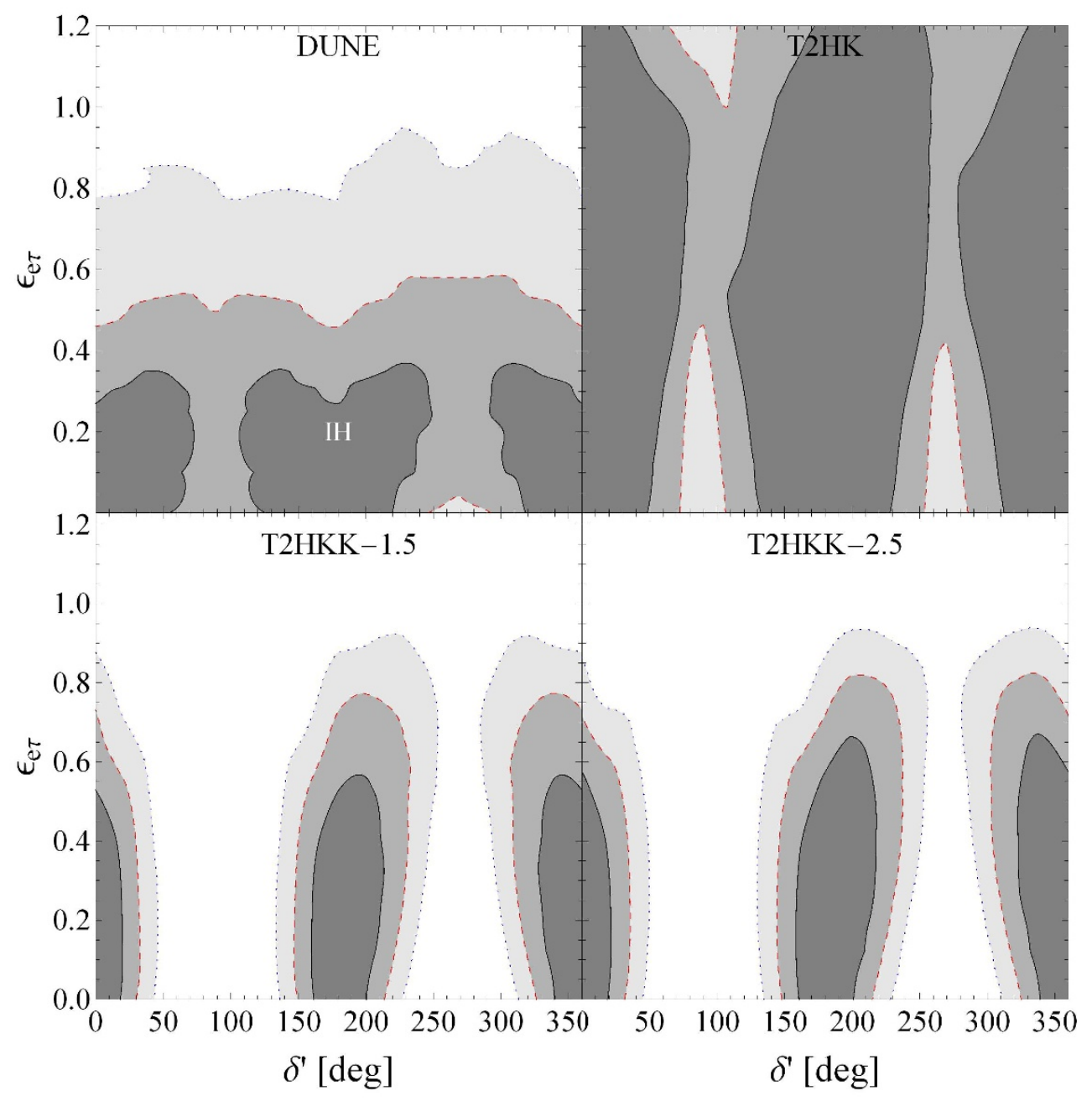

Figure 8. Same as figure 6, except for $\epsilon_{e \tau}$.

T2HKK. From figure 14 we see that there is a sharp improvement in the sensitivity to $\epsilon_{e \tau}$ at T2HKK-1.5 for $\delta \simeq 180^{\circ}$ because the IH is not allowed at the $2 \sigma$ CL in this case.

In figure 15 we show the expected sensitivities at $2 \sigma$ if $\epsilon_{e e}, \epsilon_{e \mu}$ and $\epsilon_{e \tau}$ are all nonzero and the MH is unknown. We find that the sensitivities to all three $\epsilon$ 's at T2HK and the sensitivity to $\epsilon_{e \mu}$ at T2HKK are outside the ranges that we scanned. Hence they are not shown in figure 15. If $\epsilon_{e e}, \epsilon_{e \mu}$ and $\epsilon_{e \tau}$ are all nonzero, knowledge of the mass hierarchy does not affect the sensitivity to $\epsilon_{e \mu}$ and $\epsilon_{e \tau}$ because we marginalize over the $\epsilon$ 's thereby covering the regime of the generalized MH degeneracy even if the MH is known. Furthermore, we see that the dependence on $\delta$ becomes much weaker if all three $\epsilon$ 's are nonzero, and that DUNE has the best sensitivity to the magnitude of the NSI parameters overall. An examination of our figures shows that T2HKK-1.5 has better sensitivities than T2HKK-2.5 in both the SM and NSI scenarios. 


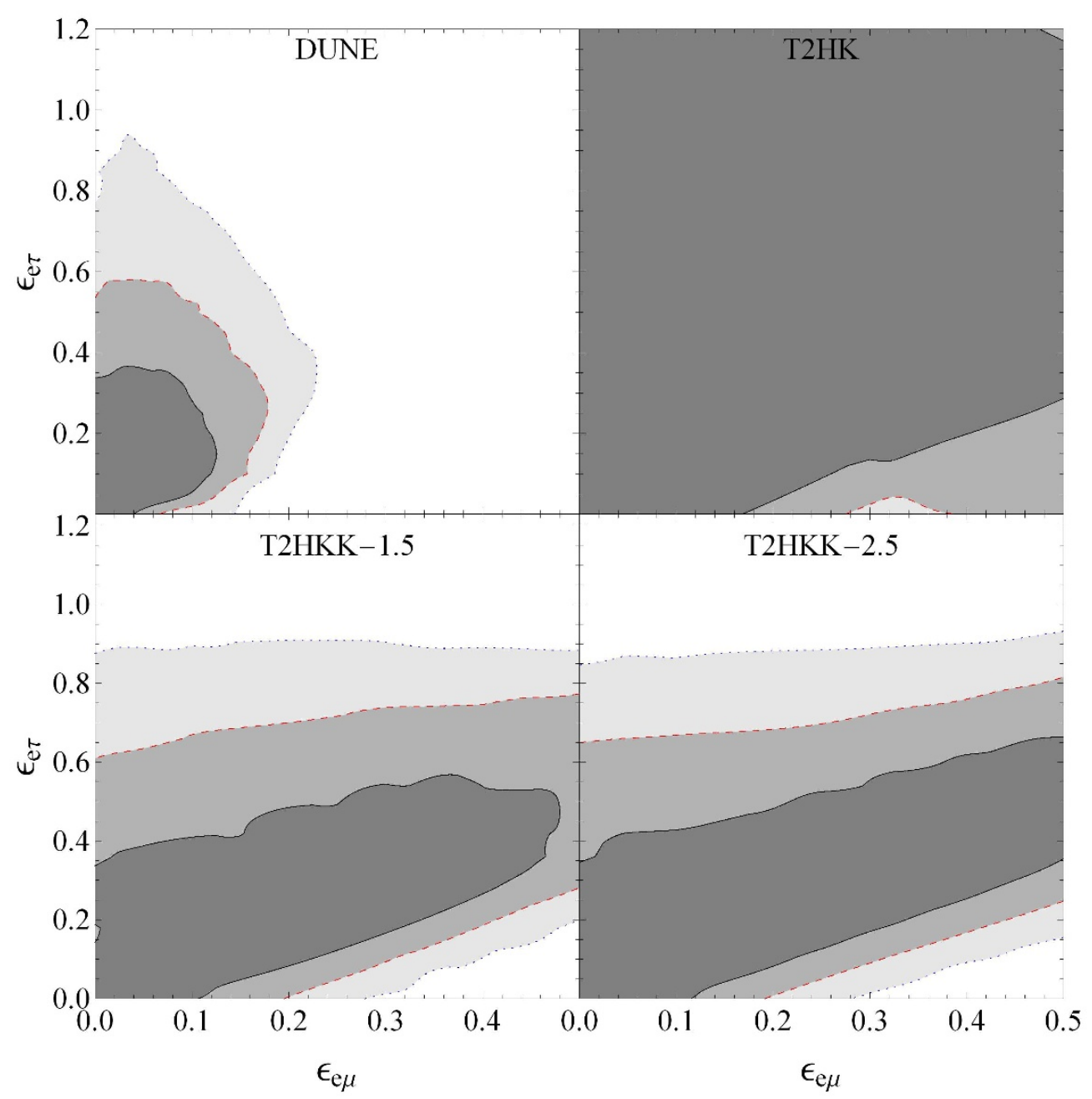

Figure 9. $1 \sigma, 2 \sigma$ and $3 \sigma$ allowed regions in the $\epsilon_{e \mu}-\epsilon_{e \tau}$ plane at DUNE, T2HK and T2HKK, assuming $\epsilon_{e e}, \epsilon_{e \mu}$ and $\epsilon_{e \tau}$ are all nonzero. The data are consistent with the SM and the NH with $\delta=0$, and all parameters not shown have been marginalized over.

\subsubsection{Sensitivity when the true mass hierarchy is inverted}

We now study the scenario in which the data are consistent with the SM with the IH. We find that there is a similarity between the allowed regions for when the data are consistent with the $\mathrm{IH}$ and the allowed regions for when the data are consistent with the $\mathrm{NH}$ after a phase transformation in $\delta$, i.e.,

$$
\delta \rightarrow \delta+180^{\circ}, \quad \delta^{\prime} \rightarrow \delta^{\prime}+180^{\circ}, \quad \phi_{\alpha \beta} \rightarrow \phi_{\alpha \beta}+180^{\circ} .
$$

An example of this similarity can be seen in figure 16, in which we show the allowed regions for $\epsilon$ as a function of $\delta^{\prime}$ at DUNE for two cases: (a) the data are consistent with the SM and the NH with $\delta=330^{\circ}$, and (b) the data are consistent with the SM and the IH with $\delta=150^{\circ}$. We see that a shift of $\delta^{\prime} \rightarrow \delta^{\prime}+180^{\circ}$, renders the allowed regions between the 


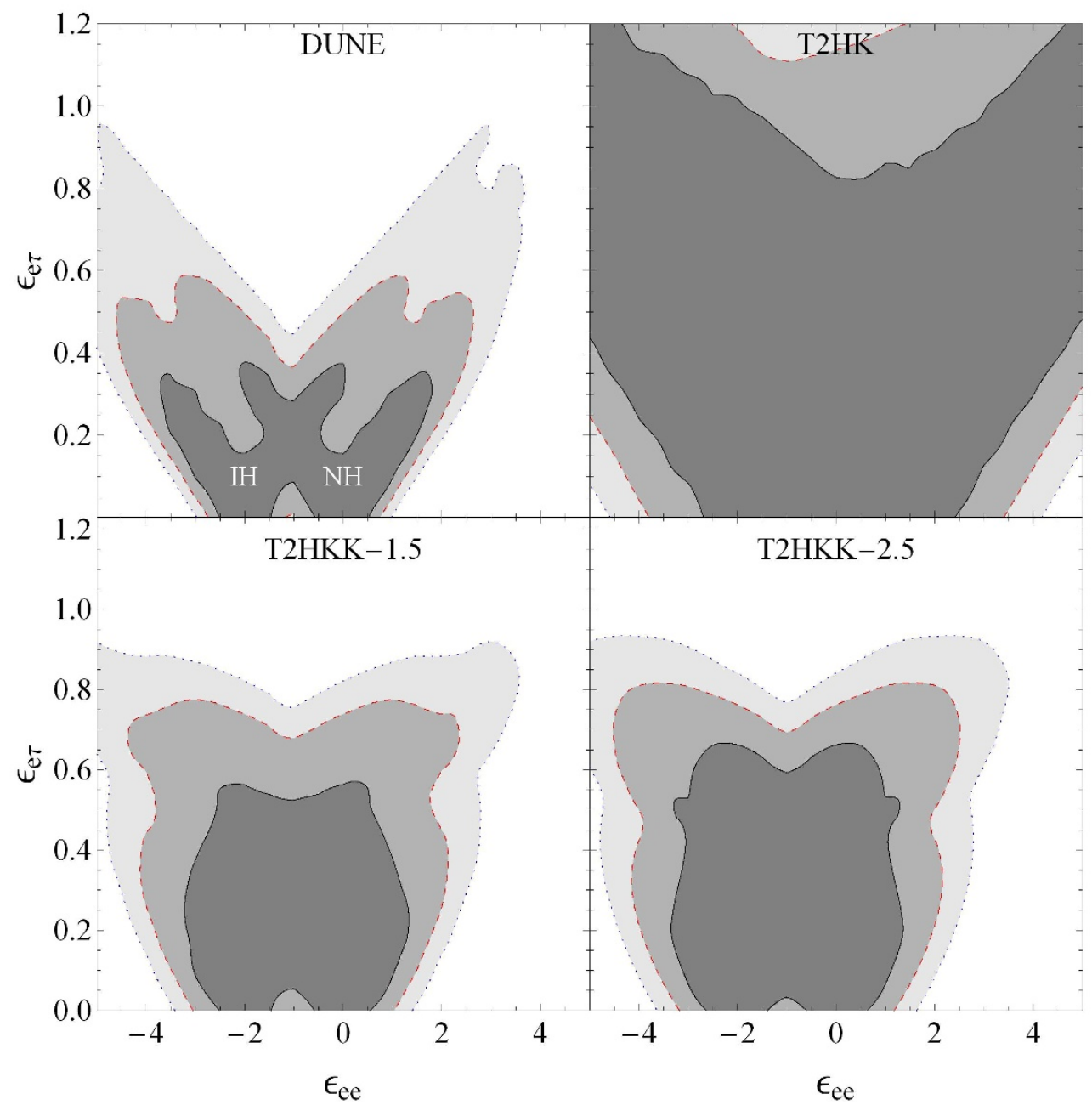

Figure 10. Same as figure 9, except for $\epsilon_{e \tau}$ versus $\epsilon_{e e}$.

two cases very similar; the allowed regions of the NSI scenario with the IH $(\mathrm{NH})$ in case (a) are similar to the allowed regions of the NSI scenario with the NH (IH) in case (b) after the phase transformation.

This similarity can be understood as follows. In order to fit the SM data with an NSI scenario, the two main constraints from the appearance channel in both the neutrino and antineutrino modes are

$$
\begin{aligned}
& P_{i}^{\mathrm{NSI}}\left(\nu_{\mu} \rightarrow \nu_{e}\right)-P_{j}^{\mathrm{SM}}\left(\nu_{\mu} \rightarrow \nu_{e}\right)=0, \\
& P_{i}^{\mathrm{NSI}}\left(\bar{\nu}_{\mu} \rightarrow \bar{\nu}_{e}\right)-P_{j}^{\mathrm{SM}}\left(\bar{\nu}_{\mu} \rightarrow \bar{\nu}_{e}\right)=0,
\end{aligned}
$$

where $i, j=\mathrm{NH}, \mathrm{IH}$. 


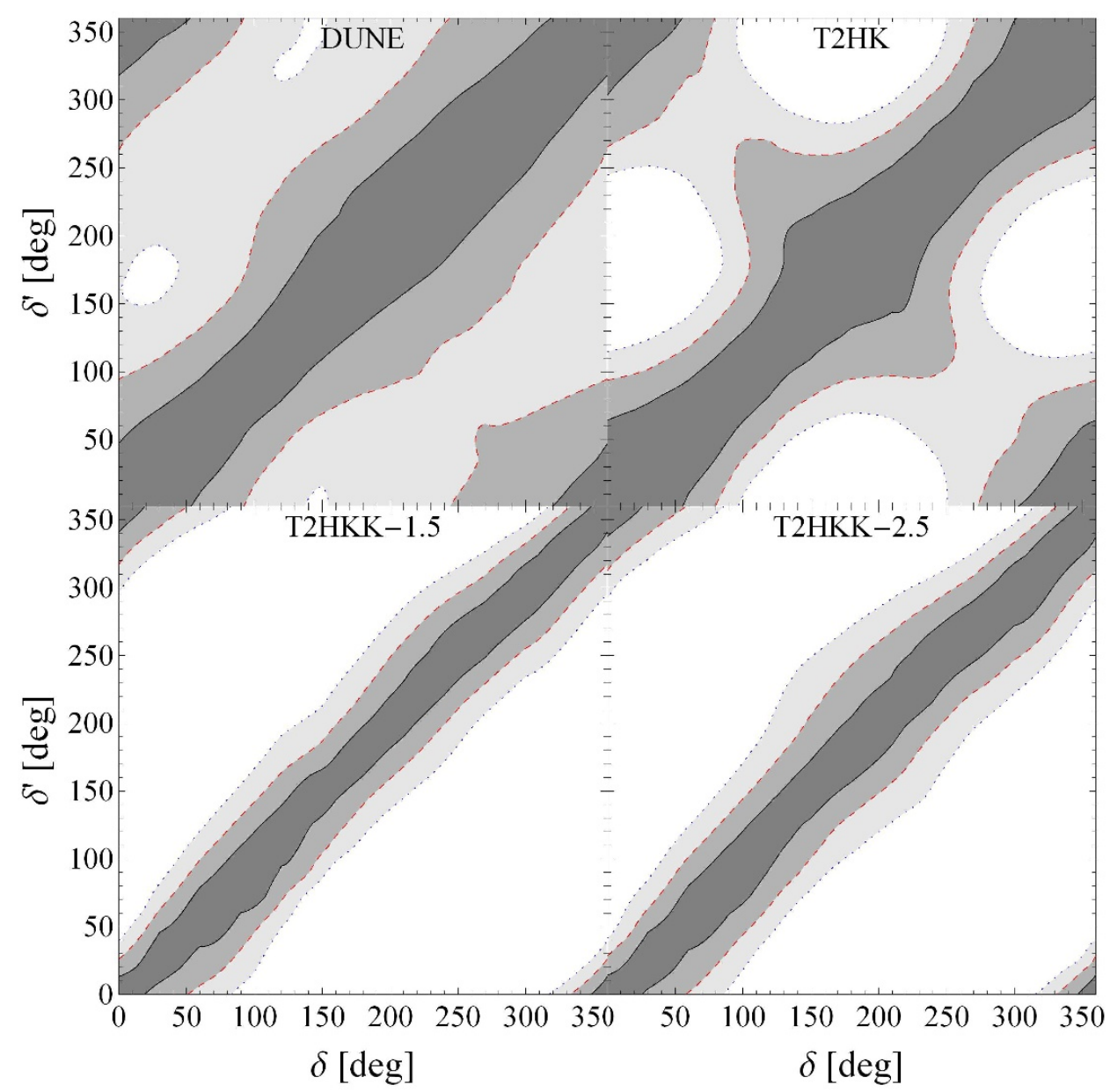

Figure 11. $1 \sigma, 2 \sigma$ and $3 \sigma$ allowed regions for $\delta^{\prime}$ as a function of $\delta$ at DUNE, T2HK and T2HKK. Here $\delta\left(\delta^{\prime}\right)$ denotes the Dirac CP phase in the SM (NSI) scenario. The data are consistent with the $\mathrm{SM}$ and the NH. We assume the mass hierarchy is known, and $\epsilon_{e e}, \epsilon_{e \mu}$ and $\epsilon_{e \tau}$ are all nonzero. All parameters not shown have been marginalized over.

Using eq. (3.1) at leading order in $\epsilon$, we have

$$
\begin{aligned}
0= & {\left[x^{2} f^{2}+2 x y f g \cos \left(\Delta+\delta^{\prime}\right)+y^{2} g^{2}\right]-\left[x^{2} \bar{f}^{2}-2 x y \bar{f} g \cos (-\Delta+\delta)+y^{2} g^{2}\right] } \\
& +4 \hat{A} \epsilon_{e \mu} x\left[s_{23}^{2} f^{2} \cos \left(\phi_{e \mu}+\delta^{\prime}\right)+c_{23}^{2} f g \cos \left(\Delta+\delta^{\prime}+\phi_{e \mu}\right)\right] \\
& +4 \hat{A} \epsilon_{e \tau} x s_{23} c_{23}\left[f^{2} \cos \left(\phi_{e \tau}+\delta^{\prime}\right)-f g \cos \left(\Delta+\delta^{\prime}+\phi_{e \tau}\right)\right], \\
0= & {\left[x^{2} \bar{f}^{2}+2 x y \bar{f} g \cos \left(\Delta-\delta^{\prime}\right)+y^{2} g^{2}\right]-\left[x^{2} f^{2}-2 x y f g \cos (-\Delta-\delta)+y^{2} g^{2}\right] } \\
& -4 \hat{A} \epsilon_{e \mu} x\left[s_{23}^{2} \bar{f}^{2} \cos \left(\phi_{e \mu}+\delta^{\prime}\right)+c_{23}^{2} \bar{f} g \cos \left(\Delta-\delta^{\prime}-\phi_{e \mu}\right)\right] \\
& -4 \hat{A} \epsilon_{e \tau} x s_{23} c_{23}\left[\bar{f}^{2} \cos \left(\phi_{e \tau}+\delta^{\prime}\right)-\bar{f} g \cos \left(\Delta-\delta^{\prime}-\phi_{e \tau}\right)\right],
\end{aligned}
$$

for the NSI scenario with the NH and the SM scenario with the IH. Switching the mass hierarchy of the SM and NSI scenarios (via $\Delta \rightarrow-\Delta, f \leftrightarrow-\bar{f}$, and $g \rightarrow-g$ ), and 


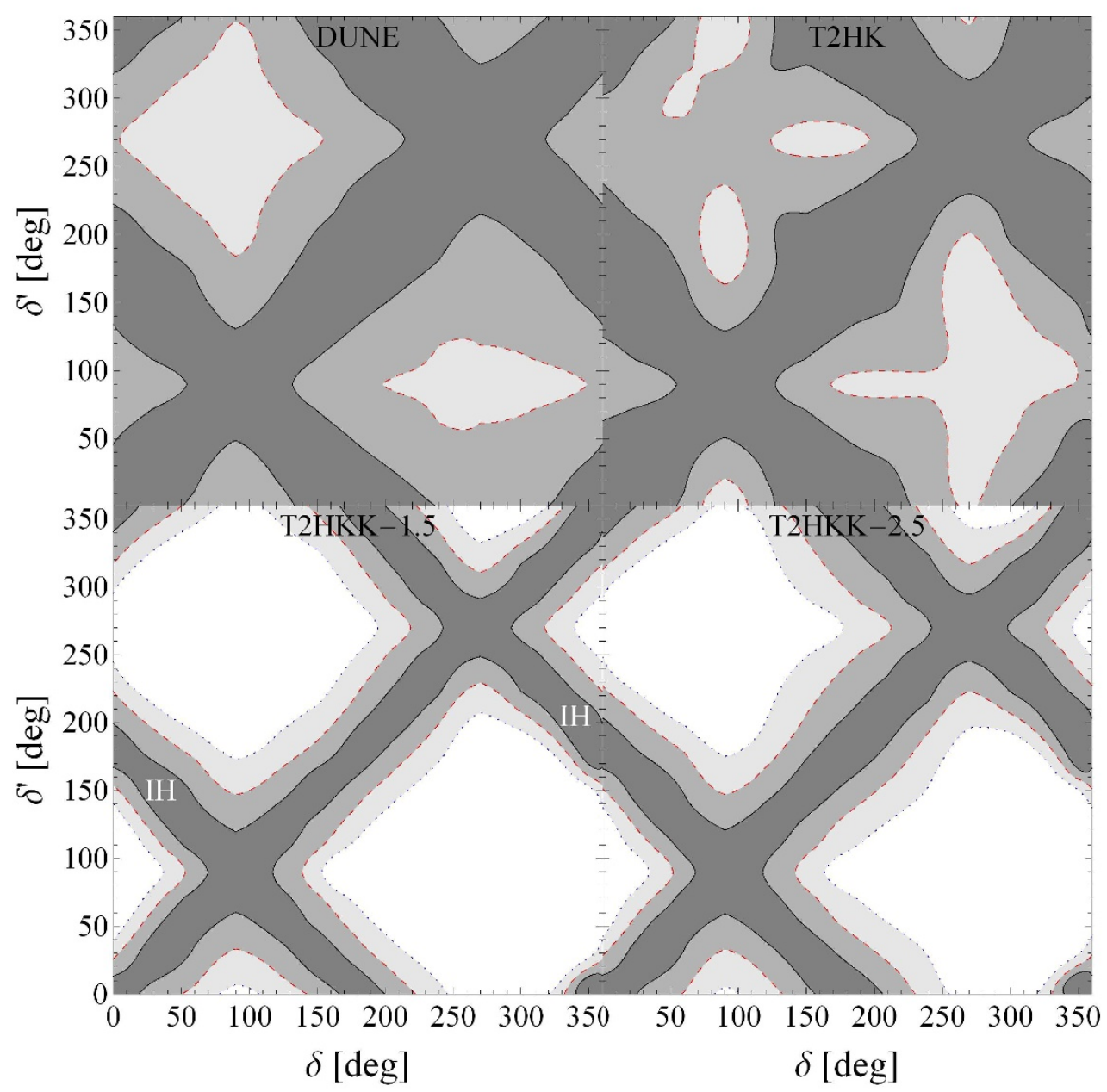

Figure 12. Same as figure 11, except that the mass hierarchy is unknown. Note that the entire $\delta-\delta^{\prime}$ parameter space is allowed at $3 \sigma$ for T2HK and DUNE.

applying the phase transformation of eq. (3.8), leads to an interchange of eq. (3.11) and eq. (3.12) so that we obtain the same two constraints on the NSI parameters. Since the phase transformation does not depend on $L$ and $E$, the allowed regions at DUNE, T2HK and T2HKK are similar for both hierarchies. However, note that if we take into account the higher order terms in eq. (3.1), in particular the third and fifth lines in eq. (3.1), the phase transformation does not leave the two constraints unchanged, which explains the small difference between the allowed regions in the two scenarios. There is a similar correspondence for any combination of hierarchies between the SM and NSI scenarios.

Because of the correspondence discussed above, we expect the NSI sensitivities to be similar whether the data are consistent with the SM in the NH or the SM in the IH. This can seen by comparing figure 15 with figure 17 . In sum, the NSI sensitivities in a given experiment will be similar regardless of the true hierarchy, modulo the transformation $\delta \rightarrow \delta+180^{\circ}$. 


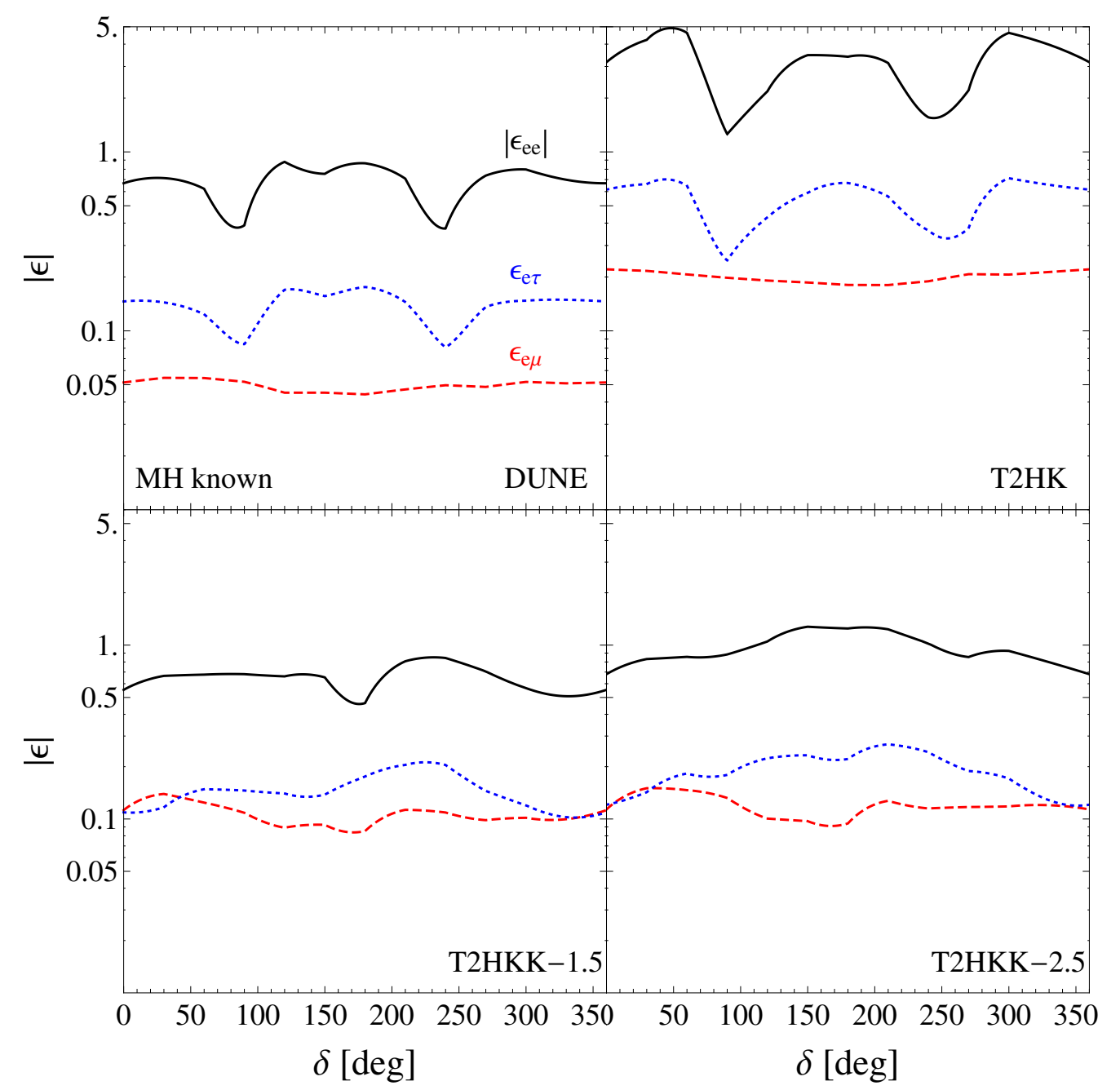

Figure 13. The expected $2 \sigma$ CL sensitivity to $|\epsilon|$ as a function of $\delta$ at DUNE, T2HK and T2HKK. The data are consistent with the SM and the NH. We assume only one $\epsilon$ is nonzero at a time and the mass hierarchy is known.

\subsection{NSI in the disappearance channels $\left(\epsilon_{\mu \tau}\right.$ and $\left.\epsilon_{\tau \tau}\right)$}

We find that the sensitivity of these experiments to $\epsilon_{\mu \tau}$ is outside the range of our scan. After marginalizing over $\epsilon_{\mu \tau}$ and the mass hierarchy, we show the $2 \sigma$ sensitivities to $\epsilon_{\tau \tau}$ at DUNE, T2HK and T2HKK in figure 18. We see that T2HKK-1.5 has better sensitivity than T2HKK-2.5 because of its higher energy spectrum and larger statistics. We also see that the $2 \sigma$ sensitivity at DUNE becomes quite weak at some $\delta$ values. This is due to a degenerate region near the boundary of the ranges that we have scanned, which was first noticed in ref. [27] and can be seen in figure 19. Since this degenerate region, which occurs because of a correlation between $\epsilon_{\tau \tau}$ and the deviation of $\theta_{23}$ from maximal mixing, is at the boundary, we also show the $90 \%$ CL sensitivity curve at DUNE in figure 18 to emphasize that the sensitivity is uniform if the degenerate region is resolved by future atmospheric data. 


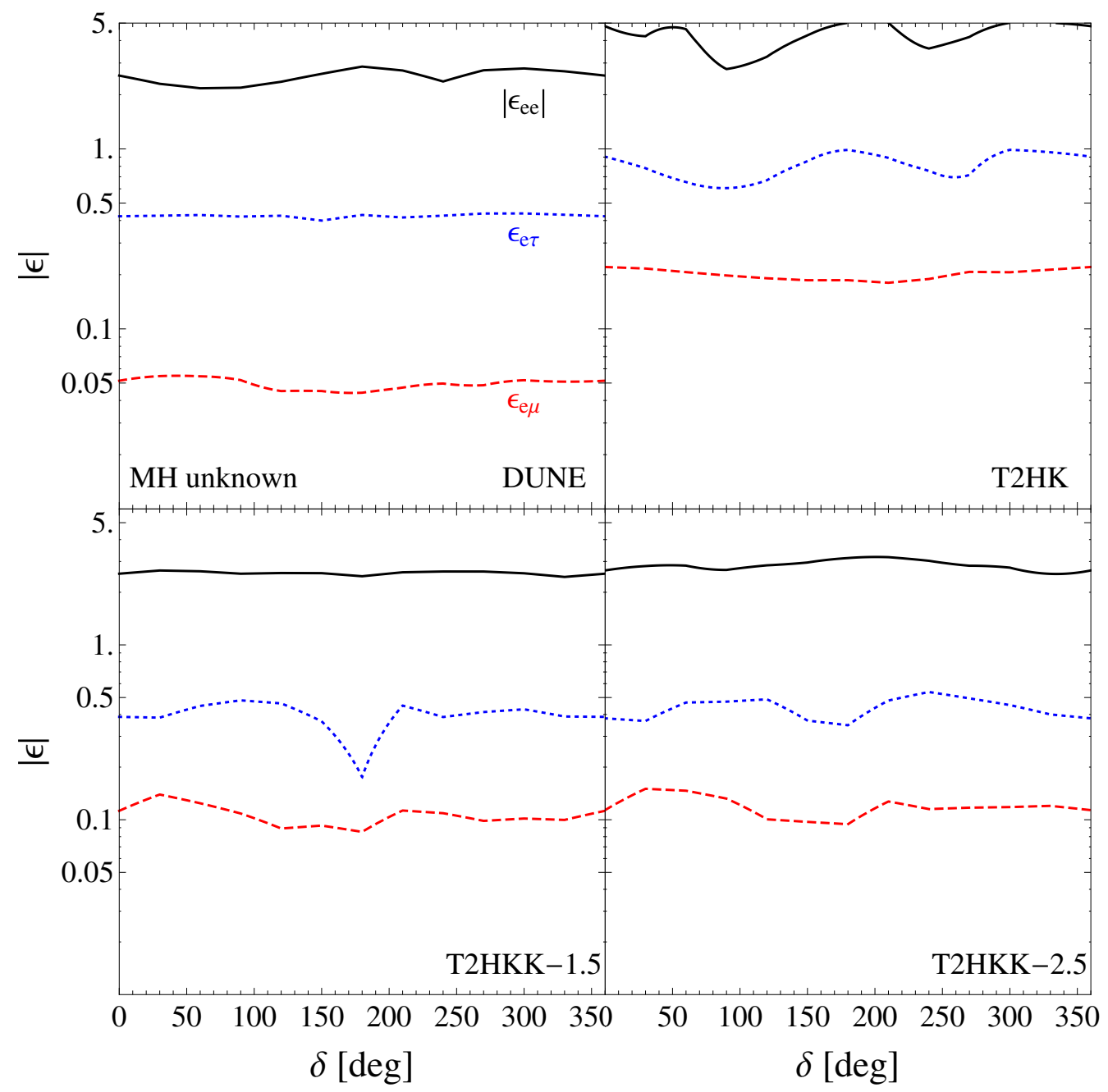

Figure 14. Same as figure 13, except that the mass hierarchy is unknown.

\section{Summary}

We studied the sensitivities to NSI in the proposed next generation long-baseline neutrino experiments DUNE, T2HK and T2HKK. For the T2HK and T2HKK experiments, we adopted the new detector configurations and fluxes provided by the Hyper-Kamiokande collaboration.

To understand the effect of each NSI parameter on the experimental performance, we considered different scenarios with different combinations of NSI parameters. We find that if only one of $\epsilon_{e \mu}$ or $\epsilon_{e \tau}$ is nonzero, most of the continuous four-fold degeneracies with NSI at a single $L$ and $E$ measurement can be resolved for the range of energies available at these experiments. The degeneracies are broken even further at T2HKK with two different baselines. However, if multiple NSI are nonzero, these experiments cannot measure the mass hierarchy, CP phase and $\theta_{23}$ octant as a result of degeneracies between NSI and SM parameters, and between NSI parameters. As a specific realization of the latter, we find 


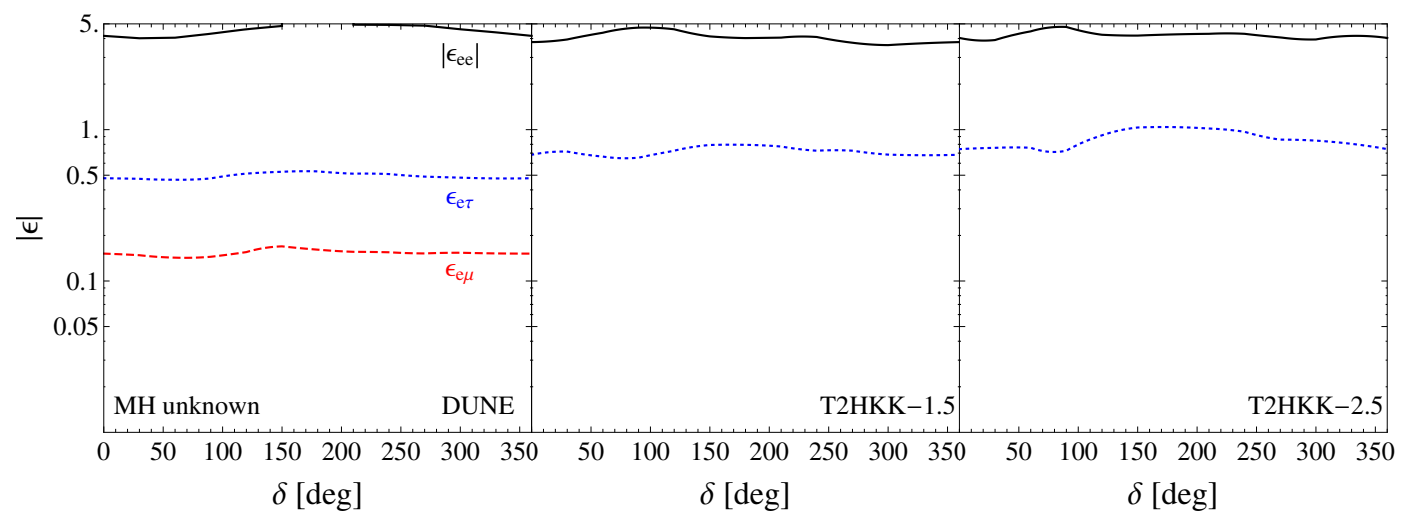

Figure 15. The expected $2 \sigma$ CL sensitivity to $|\epsilon|$ as a function of $\delta$ at DUNE and T2HKK. The data are consistent with the SM and the NH. We assume $\epsilon_{e e}, \epsilon_{e \mu}$ and $\epsilon_{e \tau}$ are all nonzero, and the mass hierarchy is unknown. For each curve, all the other parameters have been marginalized over. All sensitivities for T2HK and the $\epsilon_{e \mu}$ sensitivity for T2HKK are outside the scan range and are therefore not shown. If the mass hierarchy is known, the sensitivities are unchanged for $\epsilon_{e \mu}$ and $\epsilon_{e \tau}$ and similar for $\left|\epsilon_{e e}\right|$.

that a cancellation between terms at leading order in the appearance channel probabilities when $\epsilon_{e \tau}=\cot \theta_{23} \epsilon_{e \mu}$ strongly affects the sensitivities to these two NSI parameters at T2HK and T2HKK. Also, the sensitivities at all three experiments are worsened by the generalized mass hierarchy degeneracy in the NSI scenario. Because the generalized mass hierarchy degeneracy occurs at the Hamiltonian level, atmospheric neutrino and reactor neutrino experiments will not be able to resolve it.

We also studied the dependence of the sensitivities on the true CP phase $\delta$ and the true mass hierarchy. We find that the sensitivities are much weaker for all values of $\delta$ when multiple NSI are nonzero. Also, we find that due to leading order effects in the appearance channel probabilities, there is a similarity of the allowed regions for the NSI parameters between the case in which the data are consistent with the normal hierarchy and the case in which the data are consistent with the inverted hierarchy. Thus the sensitivities are similar whether nature has chosen the $\mathrm{NH}$ or the $\mathrm{IH}$, modulo the transformation $\delta \rightarrow \delta+180^{\circ}$.

Overall DUNE has the best sensitivity to the magnitude of the NSI parameters, while T2HKK has the best sensitivity to CP violation whether or not there are NSI, and overall T2HKK-1.5 does better than T2HKK-2.5.

We further studied the sensitivities to $\epsilon_{\mu \tau}$ and $\epsilon_{\tau \tau}$ that mainly come from the disappearance channel. We find that the sensitivities to $\epsilon_{\mu \tau}$ are limited compared to atmospheric experiments, and we obtained the sensitivity to $\epsilon_{\tau \tau}$ at these three experiments.

\section{Acknowledgments}

We thank S.-H. Seo and M. Yokoyama for useful inputs regarding T2HK and T2HKK. KW thanks the University of Hawaii at Manoa for its hospitality during part of this work. This research was supported by the U.S. DOE under Grant No. DE-SC0010504. 


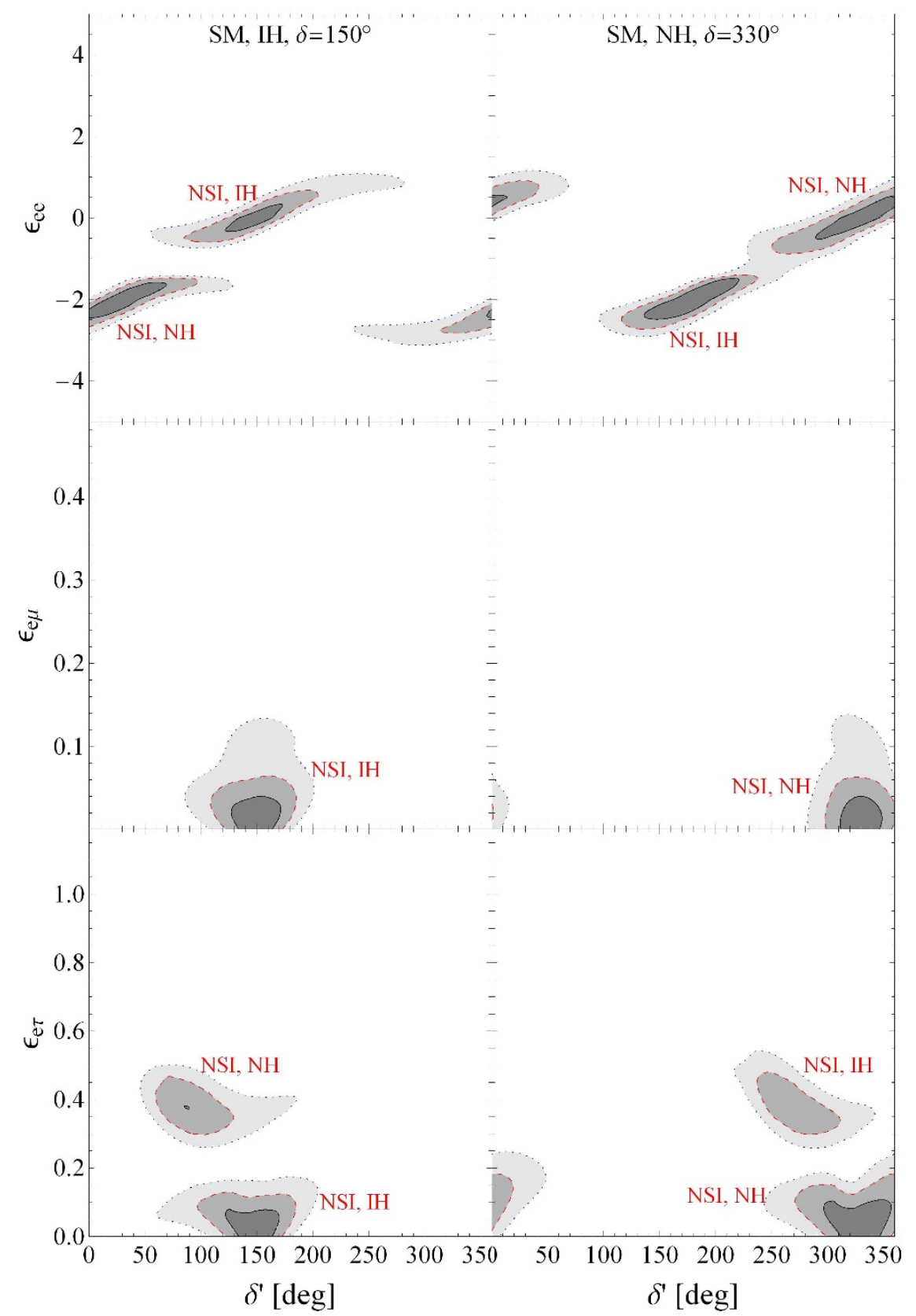

Figure 16. $1 \sigma, 2 \sigma$ and $3 \sigma$ allowed regions for $\epsilon$ as a function of $\delta^{\prime}$ at DUNE. The left (right) panels show the case when the data are consistent with the SM and the IH $(\mathrm{NH})$ with $\delta=150^{\circ}\left(330^{\circ}\right)$. Here $\delta\left(\delta^{\prime}\right)$ denotes the Dirac CP phase in the SM (NSI) scenario. We fit the data assuming only one of $\epsilon_{e e}, \epsilon_{e \mu}$ or $\epsilon_{e \tau}$ is nonzero. We scan over both mass hierarchies and marginalize over the NSI phases. 


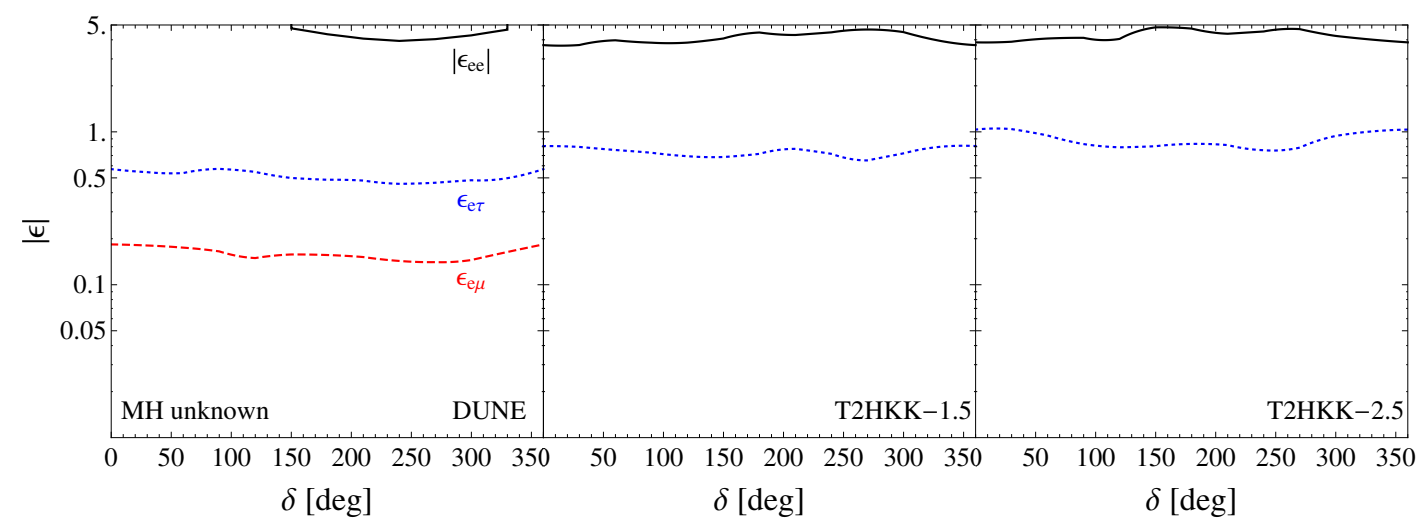

Figure 17. Same as figure 15, except that the data are consistent with the SM and the IH.

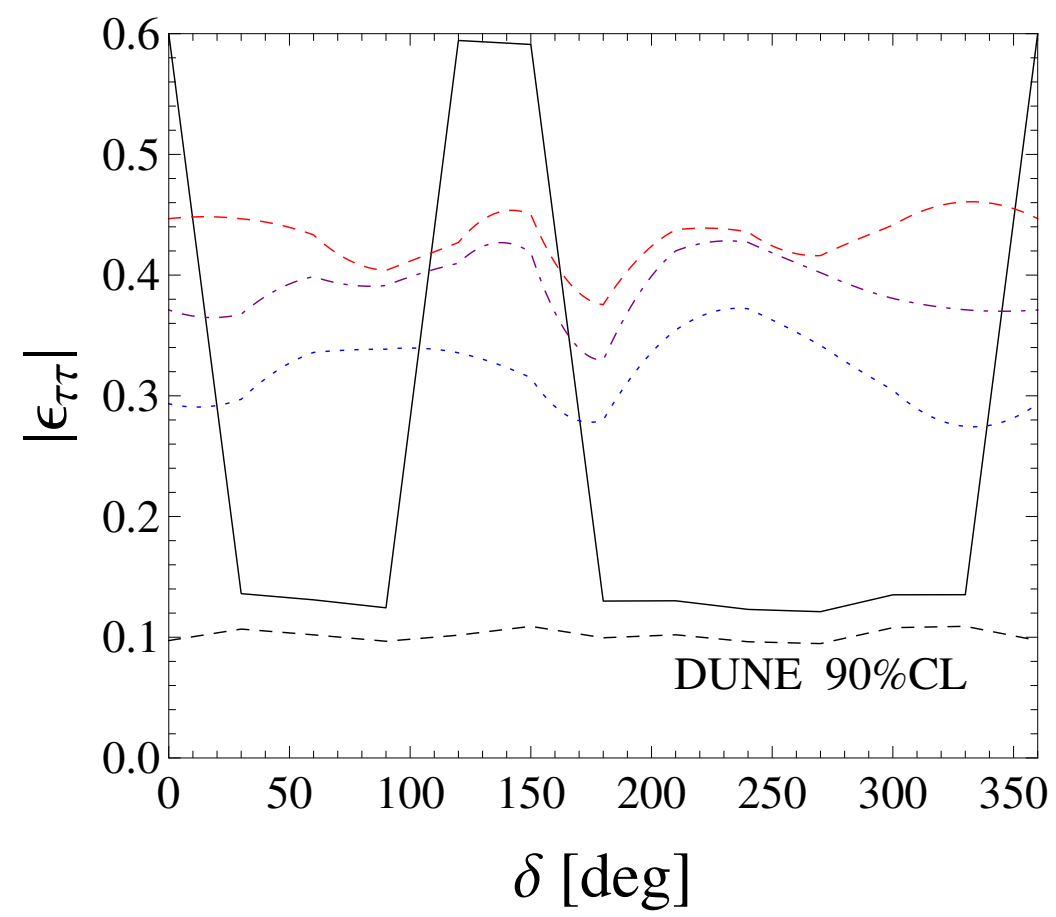

Figure 18. The expected $2 \sigma$ sensitivity to $\left|\epsilon_{\tau \tau}\right|$ as a function of $\delta$. The black solid, red dashed, blue dotted, and purple dotdashed curves correspond to DUNE, T2HK, T2HKK-1.5, and T2HKK-2.5, respectively. The data are consistent with the SM and the NH. We assume only $\epsilon_{\mu \tau}$ and $\epsilon_{\tau \tau}$ are nonzero, and all the parameters not shown have been marginalized over. We also show the $90 \% \mathrm{CL}$ sensitivity curve for DUNE to emphasize that the sensitivity is uniform if degenerate regions close to the boundaries of the scanned NSI parameter range are excluded by atmospheric data; see figure 19 for an illustration of the degenerate region. 


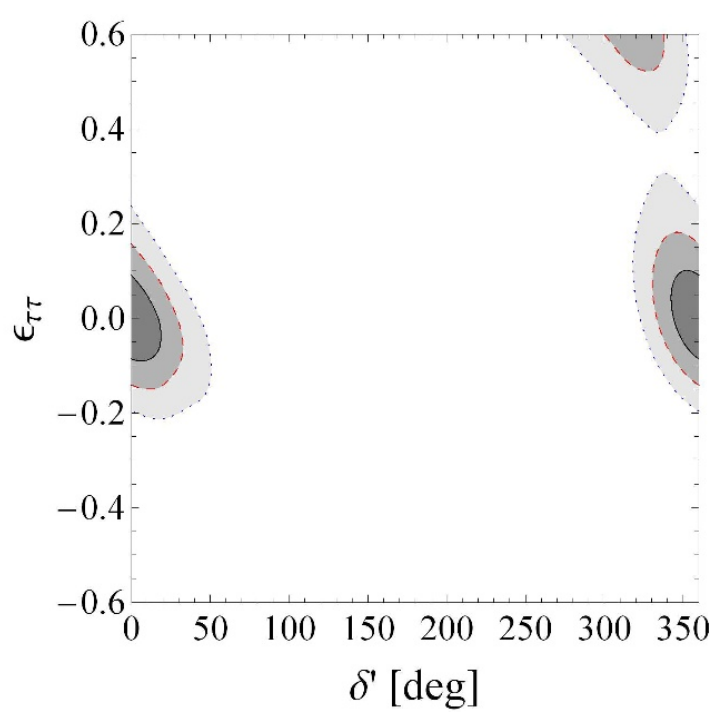

Figure 19. $1 \sigma, 2 \sigma$ and $3 \sigma$ allowed regions in the $\epsilon_{\tau \tau}$ versus $\delta^{\prime}$ plane at DUNE. The data are consistent with the SM with $\delta=0$ and the NH. We assume that only $\epsilon_{\mu \tau}$ and $\epsilon_{\tau \tau}$ are nonzero and all the parameters not shown have been marginalized over.

Open Access. This article is distributed under the terms of the Creative Commons Attribution License (CC-BY 4.0), which permits any use, distribution and reproduction in any medium, provided the original author(s) and source are credited.

\section{References}

[1] Particle Data Group collaboration, K.A. Olive et al., Review of Particle Physics, Chin. Phys. C 38 (2014) 090001 [INSPIRE].

[2] T. Ohlsson, Status of non-standard neutrino interactions, Rept. Prog. Phys. 76 (2013) 044201 [arXiv: 1209.2710] [INSPIRE].

[3] O.G. Miranda and H. Nunokawa, Non standard neutrino interactions: current status and future prospects, New J. Phys. 17 (2015) 095002 [arXiv: 1505.06254] [InSPIRE].

[4] C. Biggio, M. Blennow and E. Fernandez-Martinez, General bounds on non-standard neutrino interactions, JHEP 08 (2009) 090 [arXiv:0907.0097] [INSPIRE].

[5] L. Wolfenstein, Neutrino Oscillations in Matter, Phys. Rev. D 17 (1978) 2369 [InSPIRE].

[6] M.M. Guzzo, A. Masiero and S.T. Petcov, On the MSW effect with massless neutrinos and no mixing in the vacuum, Phys. Lett. B 260 (1991) 154 [INSPIRE].

[7] M. Blennow, T. Ohlsson and J. Skrotzki, Effects of non-standard interactions in the MINOS experiment, Phys. Lett. B 660 (2008) 522 [hep-ph/0702059] [INSPIRE].

[8] J. Kopp, P.A.N. Machado and S.J. Parke, Interpretation of MINOS data in terms of non-standard neutrino interactions, Phys. Rev. D 82 (2010) 113002 [arXiv:1009.0014] [INSPIRE].

[9] MINOS collaboration, P. Adamson et al., Search for flavor-changing non-standard neutrino interactions by MINOS, Phys. Rev. D 88 (2013) 072011 [arXiv:1303.5314] [INSPIRE]. 
[10] MINOS collaboration, P. Adamson et al., A search for flavor-changing non-standard neutrino interactions using $\nu_{e}$ appearance in MINOS, Phys. Rev. Lett. (2016)

[arXiv: 1605.06169] [INSPIRE].

[11] T2K collaboration, K. Abe et al., The T2K Experiment, Nucl. Instrum. Meth. A 659 (2011) 106 [arXiv:1106.1238] [INSPIRE].

[12] NOvA collaboration, D.S. Ayres et al., NOvA: Proposal to build a 30 kiloton off-axis detector to study $\nu(\mu) \rightarrow \nu(e)$ oscillations in the NuMI beamline, hep-ex/0503053 [INSPIRE].

[13] A. Friedland and I.M. Shoemaker, Searching for Novel Neutrino Interactions at NOvA and Beyond in Light of Large $\theta_{13}$, arXiv:1207.6642 [INSPIRE].

[14] J.A.B. Coelho, T. Kafka, W.A. Mann, J. Schneps and O. Altinok, Constraints for non-standard interaction $\epsilon_{e \tau} V_{e}$ from $\nu_{e}$ appearance in MINOS and T2K, Phys. Rev. D 86 (2012) 113015 [arXiv:1209.3757] [INSPIRE].

[15] D.V. Forero and P. Huber, Hints for leptonic CP-violation or New Physics?, Phys. Rev. Lett. 117 (2016) 031801 [arXiv:1601.03736] [INSPIRE].

[16] J. Liao, D. Marfatia and K. Whisnant, Nonmaximal neutrino mixing at NOvA from nonstandard interactions, arXiv:1609.01786 [INSPIRE].

[17] S. Fukasawa, M. Ghosh and O. Yasuda, Is nonstandard interaction a solution to the three neutrino tensions?, arXiv: 1609.04204 [INSPIRE].

[18] J. Liao, D. Marfatia and K. Whisnant, Degeneracies in long-baseline neutrino experiments from nonstandard interactions, Phys. Rev. D 93 (2016) 093016 [arXiv:1601.00927] [INSPIRE].

[19] DUNE collaboration, R. Acciarri et al., Long-Baseline Neutrino Facility (LBNF) and Deep Underground Neutrino Experiment (DUNE). Volume 2: The Physics Program for DUNE at $L B N F$, arXiv: 1512.06148 [INSPIRE].

[20] Hyper-Kamiokande Proto- collaboration, K. Abe et al., Hyper-Kamiokande, Design Report, KEK Preprint 2016-21 and ICRR-Report-701-2016-1 (2016), https://lib-extopc.kek.jp/preprints/PDF/2016/1627/1627021.pdf.

[21] Hyper-Kamiokande Proto- collaboration, K. Abe et al., Physics Potentials with the Second Hyper-Kamiokande Detector in Korea, arXiv:1611.06118 [INSPIRE].

[22] M. Masud, A. Chatterjee and P. Mehta, Probing CP-violation signal at DUNE in presence of non-standard neutrino interactions, J. Phys. G 43 (2016) 095005 [arXiv:1510.08261] [INSPIRE].

[23] A. de Gouvêa and K.J. Kelly, Non-standard Neutrino Interactions at DUNE, Nucl. Phys. B 908 (2016) 318 [arXiv:1511.05562] [INSPIRE].

[24] K. Huitu, T.J. Kärkkäinen, J. Maalampi and S. Vihonen, Constraining the nonstandard interaction parameters in long baseline neutrino experiments, Phys. Rev. D 93 (2016) 053016 [arXiv: 1601.07730] [INSPIRE].

[25] M. Masud and P. Mehta, Nonstandard interactions spoiling the CP-violation sensitivity at DUNE and other long baseline experiments, Phys. Rev. D 94 (2016) 013014 [arXiv: 1603.01380] [INSPIRE].

[26] M. Masud and P. Mehta, Nonstandard interactions and resolving the ordering of neutrino masses at DUNE and other long baseline experiments, Phys. Rev. D 94 (2016) 053007 [arXiv: 1606.05662] [INSPIRE]. 
[27] P. Coloma, Non-Standard Interactions in propagation at the Deep Underground Neutrino Experiment, JHEP 03 (2016) 016 [arXiv:1511.06357] [INSPIRE].

[28] S.K. Agarwalla, S.S. Chatterjee and A. Palazzo, Degeneracy between $\theta_{23}$ octant and neutrino non-standard interactions at DUNE, Phys. Lett. B 762 (2016) 64 [arXiv:1607.01745] [INSPIRE].

[29] H. Oki and O. Yasuda, Sensitivity of the T2KK experiment to the non-standard interaction in propagation, Phys. Rev. D 82 (2010) 073009 [arXiv: 1003. 5554] [INSPIRE].

[30] S. Fukasawa, M. Ghosh and O. Yasuda, Sensitivity of the T2HKK experiment to the non-standard interaction, arXiv: 1611.06141 [INSPIRE].

[31] P. Coloma and T. Schwetz, Generalized mass ordering degeneracy in neutrino oscillation experiments, Phys. Rev. D 94 (2016) 055005 [arXiv:1604.05772] [INSPIRE].

[32] https://wiki.hyperk.org/working-groups/wg8-beam-accelerator/fluxfiles.

[33] P. Huber, M. Lindner and W. Winter, Simulation of long-baseline neutrino oscillation experiments with GLoBES (General Long Baseline Experiment Simulator), Comput. Phys. Commun. 167 (2005) 195 [hep-ph/0407333] [INSPIRE].

[34] P. Huber, J. Kopp, M. Lindner, M. Rolinec and W. Winter, New features in the simulation of neutrino oscillation experiments with GLoBES 3.0: General Long Baseline Experiment Simulator, Comput. Phys. Commun. 177 (2007) 432 [hep-ph/0701187] [INSPIRE].

[35] DUNE collaboration, T. Alion et al., Experiment Simulation Configurations Used in DUNE $C D R$, arXiv: 1606.09550 [INSPIRE].

[36] M.C. Gonzalez-Garcia, M. Maltoni and T. Schwetz, Updated fit to three neutrino mixing: status of leptonic CP-violation, JHEP 11 (2014) 052 [arXiv:1409.5439] [INSPIRE].

[37] J. Kopp, M. Lindner, T. Ota and J. Sato, Non-standard neutrino interactions in reactor and superbeam experiments, Phys. Rev. D 77 (2008) 013007 [arXiv:0708.0152] [INSPIRE].

[38] J. Kopp, T. Ota and W. Winter, Neutrino factory optimization for non-standard interactions, Phys. Rev. D 78 (2008) 053007 [arXiv:0804.2261] [InSPIRE].

[39] A.M. Dziewonski and D.L. Anderson, Preliminary reference earth model, Phys. Earth Planet. Inter. 25 (1981) 297.

[40] M.C. Gonzalez-Garcia and M. Maltoni, Determination of matter potential from global analysis of neutrino oscillation data, JHEP 09 (2013) 152 [arXiv: 1307.3092] [INSPIRE].

[41] V. Barger, D. Marfatia and K. Whisnant, Breaking eight fold degeneracies in neutrino CP-violation, mixing and mass hierarchy, Phys. Rev. D 65 (2002) 073023 [hep-ph/0112119] [INSPIRE].

[42] T. Kikuchi, H. Minakata and S. Uchinami, Perturbation Theory of Neutrino Oscillation with Nonstandard Neutrino Interactions, JHEP 03 (2009) 114 [arXiv:0809.3312] [INSPIRE].

[43] K. Asano and H. Minakata, Large-Theta(13) Perturbation Theory of Neutrino Oscillation for Long-Baseline Experiments, JHEP 06 (2011) 022 [arXiv:1103.4387] [INSPIRE].

[44] P. Bakhti and Y. Farzan, Shedding light on LMA-Dark solar neutrino solution by medium baseline reactor experiments: JUNO and RENO-50, JHEP 07 (2014) 064 [arXiv:1403.0744] [INSPIRE].

[45] JUNO collaboration, F. An et al., Neutrino Physics with JUNO, J. Phys. G 43 (2016) 030401 [arXiv: 1507.05613] [INSPIRE]. 